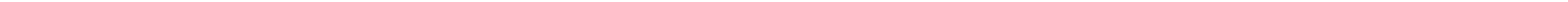




\title{
Refinement Derivatives and Values of Games
}

\author{
Luigi Montrucchio and Patrizia Semeraro ${ }^{1}$
}

February 2006

\footnotetext{
${ }^{1}$ Dipartimento di Statistica e Matematica Applicata, Università di Torino and Fondazione Collegio Carlo Alberto, Piazza Arbarello 8, 10122 Torino, Italy, Torino. E-mail: luigi.montrucchio@unito.it; URL: http://web.econ.unito.it/gma. This research was partially supported by MIUR. The authors thank Marco Scarsini for very helpful comments.

(C)2006 by Luigi Montrucchio e Patrizia Semeraro. Any opinion expressed here are those of the authors and not those of the Fondazione Collegio Carlo Alberto.
} 


\begin{abstract}
A definition of set-wise differentiability for set functions is given through refining the partitions of sets. Such a construction is closely related to the one proposed by Rosenmuller (1977) as well as that studied by Epstein (1999) and Epstein and Marinacci (2001). We present several classes of TU games which are differentiable and study differentiation rules. The last part of the paper applies refinement derivatives to the calculation of value of games. Following Hart and Mas-Colell (1989), we define a value operator through the derivative of the potential of the game. We show that this operator is a truly value when restricted to some appropriate spaces of games. We present two alternative spaces where this occurs: the spaces $p M_{\infty}$ and $P O T_{2}$. The latter space is closely related to Myerson's balanced contribution axiom.
\end{abstract}

JEL Subject classification: C71

Keywords: TU games; large games; non-additive set functions; value; derivatives 


\section{Introduction}

\subsection{Outline}

In this paper we introduce a differentiable calculus for non-additive set functions $\nu: \mathcal{A} \rightarrow$ $\mathbb{R}$ defined over an algebra $\mathcal{A}$ of sets. Special emphasis will be dedicated to TU cooperative games where $\nu$ is the characteristic function of the game. In fact, we shall make extensive use of the terminology and notation familiar in cooperative game literature.

The starting point is the definition of a set-wise derivative achieved by refining the partitions of sets. Formally, fixed a set $A$ in $\mathcal{A}$, consider a generic finite partition $\left\{H_{i}\right\}_{i=1}^{n}$ of a set $H$ disjoint from $A$. We can then look at the behavior of the sums $\sum_{i=1}^{n}\left[\nu\left(A \cup H_{i}\right)-\nu(A)\right]$ as the partitions get finer. If this refinement process has a limit for every $H$, the limit object is an additive set function $d^{+} \nu(A)(\cdot)$ over $A^{c}$, which will be regarded as the outer derivative of $\nu$ at $A$. A similar definition is obtained by means of inner increments, so leading to the inner derivative $d^{-} \nu(A)(\cdot)$. These definitions of derivatives, in which the additive set functions replace the linear functionals of the ordinary calculus for functions on vector spaces, date back to Rosenmuller [29], [30]. Though the above defined derivatives are our main objects of investigation, beside them we introduce outer and inner differentials as well. Differentials, rather than derivatives, are originated from Epstein [9] and Epstein and Marinacci [10] and rely on a stronger refinement process. Differentials are far more tractable and a more fruitful calculus, than the one reached merely by derivatives, can be carried out.

After discussing in Section 3 the basic definitions and related issues, Section 4 is devoted to the study of differentiable set functions. We show that this family is quite broad, encompassing finite games, convex games and measure games with some minor qualifications. A nontrivial result of this nature will state that any game in $p C_{\infty}$ is differentiable, having denoted by $p C_{\infty}$ the $\|\cdot\|_{\infty}$-closure of the algebra spanned by the set of all Lipschitz convex games. Section 5 is dedicated to accomplish a calculus built upon the derivatives we have introduced. The idea that a set-wise calculus, rather similar to the ordinary one, has to hold goes back to [9] and [10]. We push considerably further their original project. By Ekeland's variational principle [8] we establish an approximate

mean value theorem. Further, a general theorem on the product differentiation rule is delivered. Both these technical tools turn out to be indispensable to cope with most of the issues raised in the present paper.

While we feel that this set-wise calculus may hopefully offer an useful tool for some area of applied mathematics, the last two sections are focussed on its application in the value theory of large TU games ([1] and [26] are the best general references on this subject). The fact that derivatives matter in value theory is clearly understood, both for finite games and non-atomic games. Aumann and Shapley [1] shaped a notion 
of derivative for games with a continuum of players, by creating the $n a$-extension of games to the ideal coalitions. As this space is linear they can then perform a traditional Gateaux-like derivative. This elegant differential approach to nonatomic games leads to the so-called diagonal formula in the games space $p N A$. This way the value of a coalition finds interpretation as its marginal contribution to the typical coalitions, averaged over all such coalitions. While this method has been extended to more general spaces than $p N A$ (see [21] and [26]), the extension of games to the ideal coalitions needs some kind of non-atomiqueness for the games. Here we obviate this inconvenient by adopting the "potential" point of view undertaken for finite games by Hart and Mas-Colell [12].

In [12] the value of finite games is axiomatized by a potential function which assigns a real number to each game. Fitting their approach into our setting, this amounts to constructing a new game $u$, called the potential of $\nu$, associated with the original game $\nu$. The Shapley value of a coalition $S$ is then the marginal contribution of $S$ to the grand coalition of the potential game $u$. It is readily seen that their construction may be formulated through set-wise inner derivatives. Formally, the potential $u$ is defined implicitly by the relation $d^{-} u(A)(A)=\nu(A)$ for all $A$. Therefore, the Shapley value turns out to be $S h \nu(S)=d^{-} u(\Omega)(S)$ for all coalitions $S$, where $\Omega$ is the grand coalition.

The value approach via potentials may give rise to two possible developments for games with infinitely many players. One generalization is due to Hart and Monderer [13] who construct a similar potential theory for games having na-extensions, along Aumann and Shapley's tradition. Here we are choosing an alternative direction, somewhat closer to the original Hart and Mas-Colell's view, consisting in using our set-wise differentiable framework. This permits us to cope with mixed games where not all the players are individually negligible.

In Section 6 potential games are studied along this line. The main result is that every game in $p M_{\infty}$, the closure of the algebra spanned by finite $\sigma$-additive measures, has a potential within $p M_{\infty}$. Section 7 studies the value operator according to the marginal contribution interpretation. When restricting this operator to some appropriate spaces of games, we show that it turns out to be a truly value. We present two alternative spaces in which this occurs: the spaces $p M_{\infty}$ and $P O T_{2}$. In the former case the value coincides with the asymptotic value, so giving a "marginal" interpretation of the asymptotic value. The latter space $\mathrm{POT}_{2}$ is closely related to the second order set-wise differentiability that in turn is connected to Myerson's balanced contribution axiom.

The paper is completed by a preliminary Section containing notation and basic concepts used throughout the paper, and a final Section into which all the proofs are gathered. 


\subsection{Related literature}

The refinement process of partitions recurs frequently in many fields of measure theory, mainly for subadditive set functions (see [2]). The earliest use of the refinement limit to define derivatives is due to Rosenmuller [29], [30] with the purpose of studying the extreme points of the cone of convex games. Our derivatives $d^{+} \nu(A)$ and $d^{-} \nu(A)$ are just a direct emanation of Rosenmuller's ones. His construction is limited to convex set function and he does not develop a calculus based on them.

Epstein's definition of differential [9] comes from a decision theory context, where the set function $\nu$ is a non-additive probability. In Epstein and Marinacci [10] the same definition of differential is given with the purpose of studying the core of cooperative games. Their differential coincides with our differential $D \nu(A)$, though we do not impose the additive measure $D \nu(A)$ to be convex-ranged like [9] and [10].

Marinacci and Montrucchio [17] elaborated a subcalculus for set functions and related subderivatives to the cores of games, extending some ideas from [10].

As already mentioned, Hart and Monderer [13] extend the potential approach to differentiable non-atomic games. Their differentiability notion is closely related to a Frechet property of the differential of the na-extension of the game. They further provide an extension of their theory to the weighted case.

Finally, it is worth mentioning that the method of refining finite partition was originally introduced in the game theoretic field by Kannai [16] (see also [1] and [26]) to define the asymptotic value of large games. Even if the asymptotic method to calculate the value has some point in common with ours, a sharp link is difficult to establish. However, a closer relation will later become more transparent for games in $p M_{\infty}$.

\section{Preliminaries}

Let $\mathcal{A}$ be an algebra of subsets of a given space $\Omega$, a set function $\nu$ on $(\Omega, \mathcal{A})$ is a map $\nu: \mathcal{A} \rightarrow \mathbb{R}$. If in addition $\nu(\varnothing)=0$, the set function will be called a (coalitional) game (with side payments). In this setting, a member $\omega \in \Omega$ is a player, a set $A \in \mathcal{A}$ is viewed as a coalition and $\nu(A)$ the worth of coalition $A$. Subsets $A$ of $\Omega$ are always understood as belonging to $\mathcal{A}$, even without mentioning. When $\mathcal{A}$ is a $\sigma$-algebra, it is denoted by $\Sigma$. In some final results we need that the space $(\Omega, \Sigma)$ be a standard Borel space. As it is customary, in such a case we frequently omit $\Sigma$ in many related symbols.

The algebra $\mathcal{A}_{A}=\{B \in \mathcal{A}: B \subseteq A\}$ is the restriction of the algebra $\mathcal{A}$ to $A$. Likewise $\nu_{A}$ means the restriction of the set function $\nu$ to $\left(A, \mathcal{A}_{A}\right)$. By $\nu^{A}$ we design the set function $\nu^{A}(S)=\nu(A \cap S)$ for all $S \in \mathcal{A}$. A class $\mathcal{F}$ of games is called restrictable if $\nu \in \mathcal{F}$ implies $\nu^{A} \in \mathcal{F}$ for all $A \in \mathcal{A}$. If $\vartheta: \Omega \rightarrow \Omega$ is a measurable automorphism, define 
$\left(\vartheta^{*} \nu\right)(A)=\nu(\vartheta A)$

A (finite and measurable) partition $\pi$ of $A$ is a finite family of disjoint elements in $\mathcal{A}$ whose union is $A$. The set $\Pi(A)$ designs the totality of the partitions of $A$. If $\pi_{1}, \pi_{2} \in \Pi(A), \pi_{1} \vee \pi_{2}=\left\{C \cap D: C \in \pi_{1}, D \in \pi_{2}\right\}$. A partition $\pi_{2} \in \Pi(A)$ is a refinement of another $\pi_{1} \in \Pi(A)$, denoted by $\pi_{2} \succeq \pi_{1}$, if each member of $\pi_{1}$ is a union of members of $\pi_{2}$. $\Pi(A)$ is thus directed by the refinement relation, in that $\pi_{1} \vee \pi_{2} \succeq \pi_{1}$ and $\pi_{1} \vee \pi_{2} \succeq \pi_{2}$ for all $\pi_{1}, \pi_{2} \in \Pi(A)$.

If $\pi \in \Pi(\Omega)$ and $A \in \Sigma, \pi_{A} \in \Pi(A)$ denotes the trace of $\pi$ over $A$, i.e., $\pi_{A}=$ $\{E \cap A: E \in \pi\}$. A partition $\pi \in \Pi(\Omega)$ generates a finite sub-algebra $\mathcal{A}_{\pi}$ of $\mathcal{A}$, whose atoms are the elements of $\pi$.

$a(\mathcal{A})$ is the set of all the finitely additive measures (or charges) on $\mathcal{A}$. The subset $b a(\mathcal{A})$ denotes those bounded and $c a(\mathcal{A}) \subset b a(\mathcal{A})$ is the set of (finite) countably additive measures. If $m \in a\left(\mathcal{A}_{A}\right)$ and $n \in a\left(\mathcal{A}_{A^{c}}\right)$, the direct sum $m \oplus n \in a(\mathcal{A})$ is defined by $(m \oplus n)(E)=m(E \cap A)+n\left(E \cap A^{c}\right)$.

In Section 5 we need product measures. Given two measures $m, n \in c a(\Sigma), m \otimes n$ is the product measure on $(\Omega \times \Omega, \Sigma \otimes \Sigma)$. More specifically, we shall make use of "diagonal measures" $(m \otimes n)_{\Delta}(H)=(m \otimes n)((H \times H) \cap \Delta)$, where $\Delta$ is the diagonal set in $\Omega \times \Omega$, provided $\Delta \in \Sigma \otimes \Sigma$ (this is the case under standardness assumption). This definition extends to finitely many measures. Therefore, $\left(m_{1} \otimes m_{2} \otimes \ldots \ldots \otimes m_{n}\right)_{\Delta}$ has an obvious interpretation. We write $\otimes^{k} m=m \otimes m \otimes \ldots . . \otimes m$ ( $k$ times $)$.

Each set function $\nu$ admits a dual set function $\bar{\nu}(A)=\nu(\Omega)-\nu\left(A^{c}\right)$ for each $A$.

Several specific classes of games are used throughout the paper.

A game $\nu$ is bounded if $\sup \{|\nu(A)|: A \in \mathcal{A}\}<+\infty$. The game is monotone if $\nu(A) \leq \nu(B)$ whenever $A \subseteq B$. It is superadditive when $\nu(A \cup B) \geq \nu(A)+\nu(B)$ for all disjoint sets $A$ and $B$. A game is convex (or supermodular) if $\nu(A \cup B)+\nu(A \cap B) \geq$ $\nu(A)+\nu(B)$ for all $A$ and $B$. Given a coalition $A$, the unanimity game $u_{A}$ is the game $u_{A}(S)=1$, if $A \subseteq S$ and $u_{A}(S)=0$ elsewhere. They are monotone and convex.

An element $N \in \mathcal{A}$ is said to be a $\nu$-null set, provided $\nu(A \cup N)=\nu(A)$ for all $A \in \mathcal{A}$. The totality of the $\nu$-null sets is denoted by $\mathcal{N}(\nu)$. A set function $\nu$ is (weakly) continuous with respect to the set function $\lambda$, or $\lambda$-continuous (formally, $\nu \ll \lambda$ ), whenever $\mathcal{N}(\lambda) \subseteq \mathcal{N}(\nu)$.

The dual notion of a null set is the carrier. $S$ is a carrier of $\nu$, provided $\nu(A \cap S)=$ $\nu(A)$ for all $A \in \mathcal{A}$, i.e., $\nu=\nu^{S} . S$ is a carrier iff $\Omega \backslash S$ is $\nu$-null. A game is termed finite provided it has a finite carrier. No confusion arises by maintaining the same name for games $\nu: \mathcal{A} \rightarrow \mathbb{R}$ where $\mathcal{A}$ is a finite algebra. In this case we shall adopt the short-hand notation: $\omega \equiv\{\omega\}$, when $\omega$ is an atom of $\mathcal{A}$.

An atom of $\nu$ is an element $A \in \mathcal{A}$ such that $A \notin \mathcal{N}(\nu)$ and for all $B \subseteq A$ either 
$B \in \mathcal{N}(\nu)$ or $A \backslash B \in \mathcal{N}(\nu) . \nu$ is nonatomic if it does not have atoms

The set function $\nu$ is inner continuous at $A$, provided $A_{n} \uparrow A$ implies $\nu\left(A_{n}\right) \rightarrow \nu(A)$. It is outer continuous at $A$, if $A_{n} \downarrow A$ implies $\nu\left(A_{n}\right) \rightarrow \nu(A) . \nu$ is continuous at $A$ if it is both inner and outer continuous at $A$. This type of continuity will be also termed chain continuity in comparison to another stronger continuity introduced later.

In the space $b v(\Sigma)$ of bounded variations games (i.e., games that are difference of two monotone games, see [1]) the relation $\nu_{1} \succeq \nu_{2}$ means $\nu_{1}-\nu_{2} \in b v^{+}(\Sigma)$, namely $\nu_{1}-\nu_{2}$ is monotone. In particular, $\nu$ is called Lipschitz, if $-m \preceq \nu \preceq m$ holds for some $m \in c a^{+}(\Sigma) . \mathcal{L}(\Sigma)$ is the totality of Lipschitz games. $\mathcal{L}(\Sigma)$ can be endowed with the norm $\|\nu\|_{\infty}=\inf \left\{m(\Omega):-m \preceq \nu \preceq m, m \in c a^{+}(\Sigma)\right\} . \mathcal{L}(\Sigma)$ turns out to be a Banach space (see [22]). Following [22], for every $\nu \in \mathcal{L}(\Sigma)$, define $\nu^{*}=\wedge\{\mu \in c a(\Sigma): \mu \succeq \nu\}$ and $\nu_{*}=\vee\{\mu \in c a(\Sigma): \nu \succeq \mu\}$.

Denote by $p M_{\infty}(\Sigma) \subset \mathcal{L}(\Sigma)$ the $\|\cdot\|_{\infty}$-closure of the algebra generated by the elements in $c a(\Sigma)$. We write $p M_{\infty}$ whenever $(\Omega, \Sigma)$ is a standard Borel space. The space $\operatorname{pol} M(\Sigma)$ consists of the games $\nu=p \circ \lambda=p\left(\lambda_{1}, \ldots, \lambda_{n}\right)$ where $p(x)$ is a polynomial and $\lambda_{i} \in c a(\Sigma)$, clearly $p M_{\infty}(\Sigma)$ is the $\|\cdot\|_{\infty}$-closure of pol $M(\Sigma)$.

A vector measure is an additive map $\mu: \mathcal{A} \rightarrow \mathbb{R}^{n}$ with $\mu=\left(\mu_{1}, \ldots, \mu_{n}\right)$ where the $\mu_{i}$ are scalar charges. A vector measure is non-atomic, if $\mu(A) \neq 0$ implies the existence of some $B \subset A$ with $\mu(B) \neq 0$ and $\mu(A \backslash B) \neq 0$. A vector measure $\mu$ is called strongly continuous if for all $\varepsilon$ there is a partition $\pi \in \Pi(\Omega)$ such that $|\mu|(A) \leq \varepsilon$ for all $A \in \pi$, where $|\mu|$ is the variation measure of $\mu$. An extension of Lyapunov theorem ensures that strongly continuous additive vector measures are convex-ranged, provided $\mathcal{A}=\Sigma$, i.e., $R(\mu)=\{\mu(A): A \in \Sigma\}$ is convex (see [2]).

If $X$ is a linear space and $f: X \rightarrow \mathbb{R}, f^{\prime}(x ; h)$ denotes the directional derivative at $x$, namely,

$$
f^{\prime}(x ; h)=\lim _{t \downarrow 0} \frac{f(x+t h)-f(x)}{t} .
$$

If $X=\mathbb{R}^{n}$ and $e_{i}$ be the $i$-th unit vector of the canonical basis of $\mathbb{R}^{n}, f_{i}^{+}(x)$ and $f_{i}^{-}(x)$ denote the one-sided partial derivatives, i.e., $f_{i}^{+}(x)=f^{\prime}\left(x ; e_{i}\right)$ and $f_{i}^{-}(x)=$ $-f^{\prime}\left(x ;-e_{i}\right)$. The one-sided gradients are $\nabla^{+} f(x)=\left(f_{1}^{+}(x), \ldots, f_{n}^{+}(x)\right)$ and $\nabla^{-} f(x)=$ $\left(f_{1}^{-}(x), \ldots, f_{n}^{-}(x)\right)$. Clearly, $\nabla^{+} f(x)=\nabla^{-} f(x)$ if and only if $f$ has partial derivatives at $x$. In this case, the one-sided gradients reduce to the gradient $\nabla f(x)$. 


\section{Derivatives}

\subsection{Refinement limit}

The concept of derivative will be based on a limiting process obtained by the refinement of the partitions. We begin hence with describing this type of net convergence.

Definition 3.1. Let $(\mu, \Omega, \mathcal{A})$ be a game. For all $E \in \mathcal{A}$ set

$$
\mu_{\#}(E)=\lim _{\pi \in \Pi(E)} \sum_{E_{i} \in \pi} \mu\left(E_{i}\right) \equiv \lim _{\pi} \sum_{i=1}^{n_{\pi}} \mu\left(E_{i}\right),
$$

where the set $\Pi(E)$ of partitions of $E$ are directed by the refinement relation.

For instance, if $\Omega=\left\{\omega_{1}, \ldots, \omega_{n}\right\}$ and $\Sigma=2^{\Omega}$, then $\mu_{\#}(A)=\sum_{\omega_{i} \in A} \mu\left(\omega_{i}\right)$.

The short-hand notation $\mu \rightarrow_{\pi} \mu_{\#}$ will be frequently adopted to indicate the above limit. When writing $\mu_{\#}$, it will always be understood that the limit $\mu_{\#}(E)$ does exist and is finite for all $E$.

As pointed out by Rosenmuller [29], the refinement limit can be seen as a limit of additive measures. To see this, associate with any $\pi \in \Pi(\Omega)$ the additive measure $m_{\pi}^{\mu}$ on $\mathcal{A}_{\pi}$ such that $m_{\pi}^{\mu}(E)=\mu(E)$ for all the atoms of $\mathcal{A}_{\pi}$. Clearly $E \in \mathcal{A}_{\pi}$ for all the partitions $\pi \succeq\left\{E, E^{c}\right\}$. Therefore,

$$
\lim _{\pi \succeq\left\{E, E^{c}\right\}} m_{\pi}^{\mu}(E)=\lim _{\pi \in \Pi(E)} \sum_{E_{i} \in \pi} \mu\left(E_{i}\right)=\mu_{\#}(E) .
$$

The basic consequence of this observation is that the set function $\mu_{\#}$ is additive over $(\Omega, \mathcal{A})$. For, if $A$ and $B$ are two disjoint elements, they belong to any $\mathcal{A}_{\pi}$, with $\pi \succeq$ $\pi_{0}=\left\{A, A^{c}\right\} \vee\left\{B, B^{c}\right\}$. Hence, $\mu_{\#}(A \cup B)=\lim _{\pi \succeq \pi_{0}} m_{\pi}^{\mu}(A \cup B)=\lim _{\pi \succeq \pi_{0}} m_{\pi}^{\mu}(A)+$ $\lim _{\pi \succeq \pi_{0}} m_{\pi}^{\mu}(B)=\mu_{\#}(A)+\mu_{\#}(B)$.

A straightforward property of the refinement limit is:

$$
\left(a_{1} \nu+a_{2} \lambda\right)_{\#}=a_{1} \nu_{\#}+a_{2} \lambda_{\#}
$$

where $a_{1}, a_{2}$ are scalars. Moreover, $\mu=\mu_{\#}$ iff $\mu \in a(\mathcal{A})$. Accordingly, $\mu \rightarrow_{\pi} \mu_{\#} \Longleftrightarrow$ $\mu-\mu_{\#} \rightarrow_{\pi} 0$.

Many theorems involving limits will require a stronger convergence property than that underlying Definition 3.1. We isolate below two important qualifications. The first one is closely related to Epstein and Marinacci's [10] approach, while the second definition imposes the set-wise convergence $\mu \rightarrow_{\pi} \mu_{\#}$ to hold uniformly across the coalitions $E$. To appreciate the definition (i) below, note that the equivalence $\mu \rightarrow_{\pi} \mu_{\#} \Longleftrightarrow \mu-\mu_{\#} \rightarrow_{\pi} 0$ implies that the definition of limit may be restated as

$$
\lim _{\pi \in \Pi(E)} \sum_{E_{i} \in \pi} \mu\left(E_{i}\right)-\mu_{\#}\left(E_{i}\right)=0 .
$$


Definition 3.2. i) A game $\mu$ is said to converge absolutely to $m \in a(\mathcal{A})$, if $|\mu-m| \rightarrow_{\pi}$ 0 . That is,

$$
\lim _{\pi \in \Pi(E)} \sum_{E_{i} \in \pi}\left|\mu\left(E_{i}\right)-m\left(E_{i}\right)\right|=0,
$$

for all $E \in \mathcal{A}$.

ii) the limit $\mu \rightarrow_{\pi} \mu_{\#}$ is said to hold uniformly if for all $\varepsilon>0$ there is $\pi^{0} \in \Pi(\Omega)$ such that

$$
\left|\mu_{\#}(E)-\sum_{E_{i} \in \pi} \mu\left(E_{i}\right)\right|<\varepsilon
$$

is true for every $E \in \mathcal{A}$ and every $\pi \in \Pi(E)$ such that $\pi \succeq \pi_{E}^{0}$.

The next Proposition clarifies the relationship among the two concepts we have introduced. The statement (ii) asserts that the two specifications limit postulated in Definition 3.2 are indeed equivalent. This property will be useful and frequently utilized throughout the paper.

Proposition 3.1. i) If $|\mu-m| \rightarrow_{\pi} 0$, then $m=\mu_{\#}$. Further, to have $|\mu-m| \rightarrow_{\pi} 0$ it suffices that $|\mu-m| \rightarrow_{\pi} 0$ holds at $\Omega$.

ii) $\mu \rightarrow_{\pi} \mu_{\#}$ uniformly iff $\left|\mu-\mu_{\#}\right| \rightarrow_{\pi} 0$.

\subsection{Derivative}

We employ the definition of refinement limit to the increments of a set function. Given a set function $\nu$ (not necessarily a game) and an element $A \in \mathcal{A}$, define the outer increment game $\Delta^{+} \nu(A)(H)=\nu(A \cup H)-\nu(A)$, for $H \in \mathcal{A}_{A^{c}}$. Likewise, the inner increment game is $\Delta^{-} \nu(A)(K)=\nu(A)-\nu(A \backslash K)$, with $K \in \mathcal{A}_{A}$. The next definition is substantially due to Rosenmuller [29] who gave a similar definition at least for convex games.

Definition 3.3. The outer derivative of $\nu$ at $A$, denoted by $d^{+} \nu(A) \in a\left(\mathcal{A}_{A^{c}}\right)$, is the refinement limit, whenever it exists and is finite, of the game $\Delta^{+} \nu(A)$ on $\mathcal{A}_{A^{c}}$, namely, $\Delta^{+} \nu(A)(\cdot) \rightarrow_{\pi} d^{+} \nu(A)(\cdot)$. Likewise, the inner derivative $d^{-} \nu(A) \in a\left(\mathcal{A}_{A}\right)$ is defined by $\Delta^{-} \nu(A)(\cdot) \rightarrow_{\pi} d^{-} \nu(A)(\cdot)$ on $\mathcal{A}_{A}$. Finally, set $d \nu(A)=d^{-} \nu(A) \oplus d^{+} \nu(A) \in a(\mathcal{A})$.

For instance the inner derivative is

$$
d^{-} \nu(A)(K)=\lim _{\pi \in \Pi(K)} \sum_{K_{i} \in \pi} \nu(A)-\nu\left(A \backslash K_{i}\right) .
$$

Clearly $d^{+} \nu(A)$ and $d^{-} \nu(A)$ are uniquely defined additive set functions. It will often be convenient to work just only with one type of derivative. It suffices to use the dual set 
function $\bar{\nu}$. The relation $\Delta^{-} \nu(A)(K)=\Delta^{+} \bar{\nu}\left(A^{c}\right)(K)$ implies that $d^{-} \nu(A)=d^{+} \bar{\nu}\left(A^{c}\right)$. Likewise, $d^{+} \nu(A)=d^{-} \bar{\nu}\left(A^{c}\right)$. Accordingly, $d \nu(A)=d \bar{\nu}\left(A^{c}\right)$.

We shall make occasionally use of Dini derivatives as well. For instance:

$$
\bar{d}^{+} \nu(A)(H)=\limsup _{\pi \in \Pi(H)} \sum_{H_{i} \in \pi} \nu\left(A \cup H_{i}\right)-\nu(A) .
$$

The definition of the lower Dini derivative $\underline{d}^{+} \nu(A)(H)$ is similar. Clearly $\bar{d}^{+} \nu(A)(\cdot)$ and $\underline{d}^{+} \nu(A)(\cdot)$ are usually non-additive and extended values.

We next fortify the concept of derivative according to the type of convergence postulated in Definition 3.2.

Definition 3.4. The set function $\nu$ is called outer differentiable at $A$, if there exists an $m \in a\left(\mathcal{A}_{A^{c}}\right)$ such that $\left|\Delta^{+} \nu(A)-m\right| \rightarrow_{\pi} 0$. The element $m=D^{+} \nu(A)$ is called its outer differential. The inner differential $D^{-} \nu(A)$ is similarly defined. Finally, $D \nu(A)=$ $D^{-} \nu(A) \oplus D^{+} \nu(A)$ and $\nu$ is called differentiable at $A$, whenever both $D^{-} \nu(A)$ and $D^{+} \nu(A)$ exist.

For instance, $m=D^{+} \nu(A)$ if

$$
\lim _{\pi \in \Pi(H)} \sum_{H_{i} \in \pi}\left|\nu\left(A \cup H_{i}\right)-\nu(A)-m\left(H_{i}\right)\right|=0 .
$$

In view of Proposition 3.1, the outer differential is uniquely defined, provided it exists, and $d^{+} \nu(A)=D^{+} \nu(A)$. That Proposition provides also the condition for the derivative $d^{+} \nu(A)$ to be the differential $D^{+} \nu(A)$ : the refinement limit $\Delta^{+} \nu(A) \rightarrow_{\pi} d^{+} \nu(A)$ must hold uniformly (or, equivalently, absolutely).

It is sometimes cumbersome to handle $d^{+} \nu(A), d^{-} \nu(A)$ as their domains are not the whole space $\mathcal{A}$ but only $\mathcal{A}_{A^{c}}$ and $\mathcal{A}_{A}$, respectively. A way to remedy this, is that of extending them to $\mathcal{A}$. Set $\widetilde{d}^{+} \nu(A)(H)=d^{+} \nu(A)(H \backslash A)$ and $\widetilde{d}^{-} \nu(A)(H)=$ $d^{-} \nu(A)(H \cap A)$ for all $H \in \mathcal{A}$. Clearly, $d \nu(A)=\widetilde{d}^{+} \nu(A)+\widetilde{d}^{-} \nu(A)$. We keep in the sequel the same notation $d^{+} \nu(A)$ and $d^{-} \nu(A)$ for these extensions, if no confusion arises.

One could ask if some other interesting definition of derivative may emerge by adopting different types of increments. This is not the case. Fix $A \in \mathcal{A}$ and define the following increments for all $X \in \mathcal{A}$

$$
\begin{aligned}
& \varphi^{1}(X)=\nu(A \cup X)-\nu(A) \\
& \varphi^{2}(X)=\nu(A)-\nu(A \backslash X) \\
& \varphi^{3}(X)=\nu(A \cup X)-\nu(A \backslash X) .
\end{aligned}
$$

Proposition 3.2. The refinement limit $\varphi_{\#}^{1}$ exists iff $d^{+} \nu(A)$ exists and then $\varphi_{\#}^{1}=$ $\widetilde{d}^{+} \nu(A)$. All the same for $\varphi^{2}$ and $\varphi^{3}$ and $\varphi_{\#}^{2}=\widetilde{d}^{-} \nu(A), \varphi_{\#}^{3}=d \nu(A)$. Further, if the limits $\varphi^{i} \rightarrow_{\pi} \varphi_{\#}^{i}, i=1,2,3$ hold uniformly then the derivatives are differentials. 
Note that the difference $\nu(A \cup X)-\nu(A \backslash X)$ can be written as $\nu(A \cup F)-\nu(A)+$ $\nu(A)-\nu(A \backslash G)$ with $F \cap A=\varnothing$ and $G \subseteq A$. Therefore the case $\varphi^{3} \rightarrow_{\pi} D \nu(A)$ (absolutely) is nothing but the definition of differentiability adopted by [9] and [10], though they impose that $D \nu(A)$ is convex-ranged.

The relationship between derivatives and differentials deserves some further comments. The definition of the differential $D^{+} \nu(A)$ requires a refinement limit manifestly stronger than the one of $d^{+} \nu(A)$. Therefore, a set function may have a derivative which is not the differential at some $A$. In fact, quite surprisingly, this is a rather exceptional phenomenon and the derivative agrees with the differential in all the relevant games we meet.

The following example illustrates the above mentioned case. Let $\Omega=[0,1]$ equipped with the algebra $\mathcal{A}$ generated by the intervals. Denote by $I_{n}$ one of the intervals: $\left[0, n^{-1}\right]$, $\left(0, n^{-1}\right),\left(0, n^{-1}\right]$ or $\left[0, n^{-1}\right)$, for $n \in \mathbb{N}$. Likewise, $J_{n}$ is one of intervals: $\left[n^{-1}, 2 n^{-1}\right]$, $\left(n^{-1}, 2 n^{-1}\right),\left(n^{-1}, 2 n^{-1}\right],\left[n^{-1}, 2 n^{-1}\right)$. Define the game $\nu$ by $\nu\left(I_{n}\right)=1, \nu\left(J_{n}\right)=-1$, for all $n \geq 2$ and $\nu=0$ elsewhere. Clearly, $\nu \rightarrow_{\pi} 0$. Since any finite partition $\pi$ of $[0,1]$ admits a finer partitions including both $I_{n}$ and $J_{n}$ for some $n$, we have $\lim \sup _{\pi \in \Pi(\Omega)} \sum_{A_{i} \in \pi}\left|\nu\left(A_{i}\right)\right|=2$. Hence, $d^{+} \nu(\varnothing)=0$ and $D^{+} \nu(\varnothing)$ does not exist.

We close this section by collecting a few useful results on the outer derivatives, provided the set function exhibits some specific characteristic. Similar results hold for inner derivatives.

Proposition 3.3. i) If $\nu$ is bounded then $D^{+} \nu(A) \in b a\left(\mathcal{A}_{A^{c}}\right)$.

ii) if $\nu$ is outer continuous at $A$, then $D^{+} \nu(A)$ is countably additive.

iii) $d^{+} \nu(A)=d^{+} \nu(B)$ whenever $A \triangle B$ is $\nu$-null.

iv) $d^{+} \nu(A) \ll \nu$ for all $A$.

v) $\nu$ is nonatomic, provided $d^{+} \nu(\varnothing)$ is nonatomic.

\section{Differentiable games}

This section is dedicated to the study of the properties of differentiability for a few important classes of games.

Any finite game is everywhere differentiable. If $(\nu, \Omega, \Sigma)$ is a game, with $\Omega=$ $\left\{\omega_{1}, \ldots, \omega_{n}\right\}$ and $\Sigma=2^{\Omega}$, we have

$$
D \nu(A)\left(\omega_{i}\right)=\nu\left(A \cup \omega_{i}\right)-\nu\left(A \backslash \omega_{i}\right)
$$

for all $\omega_{i}$. It is interesting to relate this derivative to the Owen's multilinear extension 
of a finite game (see [28]), given by the function

$$
B_{\nu}(x)=\sum_{A \in \Sigma} \nu(A) \prod_{i \in A} x_{i} \prod_{j \in A^{c}}\left(1-x_{j}\right)
$$

defined over $\mathbb{R}^{|\Omega|}$. The relation

$$
D \nu(A)\left(\omega_{i}\right)=\partial B_{\nu}\left(e_{A}\right) / \partial x_{i}
$$

holds, where $e_{A}$ is the incidence vector: $\left(e_{A}\right)_{j}=1$ if $\omega_{j} \in A$ and $\left(e_{A}\right)_{j}=0$ if $\omega_{j} \notin A$.

Convex games are related to convex functions ${ }^{1}$. It is therefore not striking that they enjoy differentiability properties of some degree, rather analogous to the ones known in convex analysis. It is convenient to introduce subderivatives too. For a given coalition $A$, the subdifferential $\partial \nu(A)$ is

$$
\partial \nu(A)=\{m \in a(\mathcal{A}): \nu(X) \geq \nu(A)+m(X)-m(A), \forall X\}
$$

A charge $m \in \partial \nu(A)$ will be called a subderivative of $\nu$ at $A$. We can also conceive the outer subdifferential $\partial^{+} \nu(A)$ as well as the inner subdifferential $\partial^{-} \nu(A)$ whose definitions are obvious. For instance, $m \in \partial^{+} \nu(A)$ if $\nu(X) \geq \nu(A)+m(X)-m(A)$ for all $X \supseteq A$.

Theorem 4.1. Let $\nu$ be a convex game. $\nu$ is outer differentiable at any $A$ if and only if $\partial \nu(\varnothing) \neq \varnothing$. Analogously, $\nu$ is inner differentiable if and only if $\partial \nu(\Omega) \neq \varnothing$. Further, under the conditions $\partial \nu(\varnothing) \neq \varnothing$ and $\partial \nu(\Omega) \neq \varnothing$, we have:

i)

$$
D^{+} \nu(A) \in \partial^{+} \nu(A), \quad D^{-} \nu(A) \in \partial^{-} \nu(A)
$$

ii)

$$
\begin{aligned}
& D^{+} \nu(A)=\vee\left\{m: m \in \partial^{+} \nu(A)\right\} \\
& D^{-} \nu(A)=\wedge\left\{m: m \in \partial^{-} \nu(A)\right\} .
\end{aligned}
$$

iii) $\nu$ is everywhere subdifferentiable (i.e., $\partial \nu(A) \neq \varnothing$ for all $A$ ). In particular, $D^{-} \nu(A) \oplus$ $\left[D^{+} \nu(\varnothing)\right]_{A^{c}} \in \partial \nu(A)$

iv) if $A \subseteq B$, then $D \nu(A) \leq D \nu(B)$.

Formulas (4.3) are somewhat similar to Moreau-Rockafellar maximum formula of convex analysis (see for instance Th. 3.1.8 of [3]) and clearly the converse formula holds as well. For example, $\partial^{+} \nu(A)=\vee\left\{m: m \leq D^{+} \nu(A)\right\}$.

\footnotetext{
${ }^{1}$ In fact, this assertion is not completely exact. Convex set functions are closely related to the class of "ultramodular" functions. They have been the object of the study [19].
} 
Theorem 4.1 rests on the fact that the outer difference $\Delta^{+} \nu(A)(\cdot)$ is superadditive, whenever the game is convex, while $\Delta^{-} \nu(A)(\cdot)$ is subadditive. It is easy to see that such properties are characteristic for convex games. There is therefore no hope of extending Theorem 4.1 to other families of set functions.

The condition of differentiability $\partial \nu(\varnothing) \neq \varnothing$ and $\partial \nu(\Omega) \neq \varnothing$ for convex games has another interesting implication, as shown in the following proposition.

Proposition 4.1. A convex game $\nu$ is Lipschitz iff $\nu$ is continuous, $\partial \nu(\varnothing) \neq \varnothing$ and $\partial \nu(\Omega) \neq \varnothing$.

The next theorem provides an important closure property for the space $\mathcal{L}(\Sigma)$ of Lipschitz games.

Theorem 4.2. The limit in $\mathcal{L}(\Sigma)$ of differentiable games is differentiable. More specifically, let $\nu_{n} \in \mathcal{L}(\Sigma)$ and $\left\|\nu_{n}-u\right\|_{\infty} \rightarrow 0$ as $n \rightarrow \infty$. If $d^{+} \nu_{n}(A)$ exist for all $n$, then $d^{+} u(A)$ exists and $d^{+} \nu_{n}(A)(H) \rightarrow d^{+} u(A)(H)$ uniformly over $H$. Further, if $d^{+} \nu_{n}(A)=D^{+} \nu_{n}(A)$ then $d^{+} u(A)=D^{+} u(A)$. The same property holds for inner derivatives.

By this theorem we can identify a rather large class of differentiable games. Let $p C_{\infty}(\Sigma)$ be the games which lie in the $\|\cdot\|_{\infty}$-closure of the algebra spanned by Lipschitz convex games. Note that $p M_{\infty}(\Sigma) \subseteq p C_{\infty}(\Sigma)$.

Proposition 4.2. Any game $\nu \in p C_{\infty}(\Sigma)$ is everywhere differentiable.

We turn now to the measure games $\nu(A)=f(\mu(A))$.

Proposition 4.3. Let $\nu=f \circ \mu$ be a measure game with $\mu=\left(\mu_{i}\right)_{i=1}^{n}$ strongly continuous positive charges. Let $\mu(A)=\bar{x}$. Under the conditions:

i) $f$ is locally Lipschitz continuous at $\bar{x}$;

ii) the directional derivative $f^{\prime}(\bar{x} ; h)$ is linear on $\mathbb{R}_{+}^{n}$, i.e., $f^{\prime}(\bar{x} ; h)=$

$\nabla^{+} f(\bar{x}) \cdot h$ for $h \geq 0$;

the measure game $\nu$ is outer differentiable at $A$ and $D^{+} \nu(A)=\nabla^{+} f(\mu(A)) \cdot \mu_{A^{c}}$. Similarly, $D^{-} \nu(A)=\nabla^{-} f(\mu(A)) \cdot \mu_{A}$, provided $f^{\prime}(\bar{x} ; h)=\nabla^{-} f(\bar{x}) \cdot h$ for $h \leq 0$.

It follows $D \nu(A)=\nabla f(\mu(A)) \mu$, provided $\nabla^{+} f(\bar{x})=\nabla^{-} f(\bar{x})$. The hypotheses of Proposition 4.3, under which the measure game is differentiable, are by no means necessary. Consider for instance the scalar measure game $\nu=f \circ \mu$, where $f$ is a discontinuous solution to the Cauchy equation $f(x+y)=f(x)+f(y)$. Clearly, $D \nu(A)=f \circ \mu$ for all $A \in \mathcal{A}$.

The hypothesis of positivity of $\mu$ in this Proposition can be dispensed with. It suffices to assume that $f^{\prime}(\bar{x} ; \cdot)$ is linear on $\mathbb{R}^{n}$ and the proof goes through almost identically. 
Specializing the measure games $f(\mu(A))$, the conclusion of Proposition 4.3 holds true under much weaker assumptions on the function $f$, although we need measures in place of charges. Unlike Proposition 4.3, in the next statement the directional derivative $f^{\prime}(\bar{x} ; \cdot)$ is not assumed to be linear.

Proposition 4.4. Let $\nu=f \circ \mu$ and $\mu=\left(\mu_{1}, \mu_{2}, \ldots, \mu_{n}\right)$ be non-atomic and positive measures which are mutually singular and $\Sigma$ be a $\sigma$-algebra. If $\mu(A)=\bar{x}$, and $\nabla^{+} f(\bar{x})$ exists, then $D^{+} \nu(A)=\nabla^{+} f(\mu(A)) \cdot \mu_{A^{c}}$. Likewise, $D^{-} \nu(A)=\nabla^{-} f(\mu(A)) \cdot \mu_{A}$.

To illustrate this last result, we calculate the derivatives of the glove market game $\nu(A)=\min _{i=1,2, \ldots, n} \mu_{i}(A)$, namely, the minimum of a finite number of mutually singular measures $\mu_{i} \in n a^{+}(\Sigma)$. Proposition 4.3 does not help us since the directional derivative is not linear at many points. We can yet exploit Proposition 4.4. By computing the directional derivative of $f(x)=\min _{j=1, . ., n} x_{j}$, (see for instance [3, Prop. 2.3.2]), we get easily:

$$
\begin{aligned}
D^{-} \nu(A) & =\sum_{i \in \Gamma(\mu(A))} \mu_{i} \\
D^{+} \nu(A) & =\left\{\begin{array}{ll}
0 & \text { if } \Gamma(\mu(A)) \text { is not a singleton } \\
\mu_{i} & \text { if } \Gamma(\mu(A))=\{i\}
\end{array} .\right.
\end{aligned}
$$

where $\Gamma(x)=\left\{i \in\{1, \ldots, n\}: x_{i}=\min _{j=1, \ldots, n} x_{j}\right\}$.

\section{Calculus}

One of the most important applications of the classical differential calculus is the provision of derivative criteria for various properties of functions such as monotonicity, convexity and so on. In in this section corresponding criteria for set functions are discussed. Some additional regularity assumptions on the set functions as well as on the structure on the space $(\Omega, \mathcal{A})$ are needed, otherwise the behavior of the set function is rather unrelated to its derivatives. The next definitions of continuity for set functions are necessary to formulate a general approximate mean value theorem.

Definition 5.1. i) A set function $\nu$ is upper semicontinuous in measure if there exists $m \in c a^{+}(\Sigma)$ such that $\nu \ll m$ and, given $\left\{A_{n}\right\}_{n=1}^{\infty}$ and $A$ in $\Sigma$, if $m\left(A_{n} \triangle A\right) \rightarrow 0$ then $\nu(A) \geq \lim \sup _{n} \nu\left(A_{n}\right)$ (similar definition for lower semicontinuity);

(ii) $\nu$ is continuous in measure if for some $m \in c a^{+}(\Sigma), m\left(A_{n} \triangle A\right) \rightarrow 0$ implies $\nu\left(A_{n}\right) \rightarrow \nu(A)$.

If $\nu$ is continuous in measure, then $\nu$ is chain continuous. Further, $\nu \ll m$. The measure $m$ in Definition 5.1 will be called a control measure in the sequel. 
The next Proposition shows that the measure-continuous set functions consist of a rather large class, containing relevant games. Denote by $\mathcal{C}(\Sigma)$ the class of the bounded and measure-continuous set functions.

Proposition 5.1. $\mathcal{C}(\Sigma)$ is an algebra, closed by uniform limits. Further, it contains: i) all chain-continuous exact games (see [32]), in particular, any continuous and bounded convex game,

ii) the class $p N A^{\prime}$ of all games having na-continuous extensions to the space of ideal coalitions, provided $(\Omega, \Sigma)$ is a standard Borel space,

iii) any Lipschitz game, i.e., $\mathcal{L}(\Sigma) \subset \mathcal{C}(\Sigma)$,

$i v)$ all the measure games $f(\mu(A))$, where $f$ is continuous on the range $R(\mu)$ of a vector measure $\mu$, not necessarily non-atomic,

v) any finite game and any chain-continuous game on $\left(\mathbb{N}, 2^{\mathbb{N}}\right)$ of bounded variation.

The identity $A \triangle B=A^{c} \triangle B^{c}$ implies that $\mathcal{C}(\Sigma)$ is closed by conjugation.

It is apparent in the proof of point (i) of this Proposition that the measure-continuity is equivalent to a true continuity for functions defined over an appropriate metric space (more precisely, the metric probability space $\Sigma(m)$ associated with the measure space $(\Omega, \Sigma, m)$, see the proof of this proposition). Accordingly, the sequential definition of continuity can be reformulated as: for all $\varepsilon>0$, there is $\delta>0$ such that $m(B \triangle A) \leq$ $\delta \Longrightarrow|\nu(B)-\nu(A)| \leq \varepsilon$.

We are in a position to formulate the above mentioned mean value theorem. Its proof relies on Ekeland's variational principle [8].

Theorem 5.1. Under the assumptions:

i) $\Sigma$ is a $\sigma$-algebra,

ii) $\nu$ is bounded from above and upper semicontinuous in measure, with control measure $m$,

iii) $d^{+} \nu(E)$ exists for all $E \in \Sigma$,

iv) $A \subseteq B$, with $m(A) \neq m(B)$,

then, for all $\varepsilon>0$ sufficiently small, there is a set $C_{\varepsilon}$, with $A \subseteq C_{\varepsilon} \subseteq B$ and $m\left(C_{\varepsilon}\right) \neq$ $m(B)$, and a scalar $\alpha_{\varepsilon} \in[k, 1]$, with $k>0$, independent of $\varepsilon$, so that

$$
d^{+} \nu\left(C_{\varepsilon}\right)\left(B \backslash C_{\varepsilon}\right) \leq \alpha_{\varepsilon}[\nu(B)-\nu(A)]+\varepsilon^{1 / 2} m\left(B \backslash C_{\varepsilon}\right) .
$$

If in addition $d^{-} \nu(E)$ exists for all $E$, then

$$
d^{-} \nu\left(C_{\varepsilon}\right)\left(C_{\varepsilon} \backslash A\right) \geq\left(1-\alpha_{\varepsilon}\right)[\nu(B)-\nu(A)]-\varepsilon^{1 / 2} m\left(C_{\varepsilon} \backslash A\right) .
$$

Alternatively, one can replace in (5.1) and (5.2) $\alpha_{\varepsilon}$ by $\beta_{\varepsilon} \in\left[0, k^{\prime}\right]$, with $k^{\prime}<1$ and $m\left(C_{\varepsilon}\right) \neq m(A)$. 
Replacing $\nu$ by $-\nu$ we get a similar statement for lower semicontinuous set functions. Equations (5.1) and (5.2) change into

$$
\begin{aligned}
& d^{-} \nu\left(\bar{C}_{\varepsilon}\right)\left(\bar{C}_{\varepsilon} \backslash A\right) \leq\left(1-\alpha_{\varepsilon}\right)[\nu(B)-\nu(A)]+\varepsilon^{1 / 2} m\left(\bar{C}_{\varepsilon} \backslash A\right) \\
& d^{+} \nu\left(\bar{C}_{\varepsilon}\right)\left(B \backslash \bar{C}_{\varepsilon}\right) \geq \alpha_{\varepsilon}[\nu(B)-\nu(A)]-\varepsilon^{1 / 2} m\left(B \backslash \bar{C}_{\varepsilon}\right)
\end{aligned}
$$

for some $A \subseteq \bar{C}_{\varepsilon} \subseteq B$.

We draw some immediate consequences. In the next two propositions we shall assume without mentioning that the games are bounded and defined on a $\sigma$-algebra $\Sigma$.

Proposition 5.2. Let $\nu$ have outer derivative for all $A \in \Sigma$. $\nu$ is monotone if and only if $d^{+} \nu(A) \geq 0$ for all $A$, provided $\nu$ is upper semicontinuous in measure.

The condition $d^{-} \nu(A) \geq 0$ leads to a similar statement for lower semicontinuous set functions. Another important implication is the following result which will play an important role in the next Section.

Proposition 5.3. If $\nu_{1}, \nu_{2} \in \mathcal{C}(\Sigma)$, then $\nu_{1}-\nu_{2}=$ const iff $d^{+} \nu_{1}(A)\left(A^{c}\right)=d^{+} \nu_{2}(A)\left(A^{c}\right)$ for all $A$; equivalently, iff $d^{-} \nu_{1}(A)(A)=d^{-} \nu_{2}(A)(A)$ for all $A$.

Consider the measure game $f_{q} \circ \mu$ with $\mu \in n a^{1}(\Sigma), q \in(0,1), f_{q}(x)=1$ if $x \geq q, f_{q}(x)=0$ if $x \in[0, q)$. Clearly $\nabla^{+} f_{q}(x)=0$ for all $x$. By Proposition 4.4, $D^{+}\left(f_{q} \circ \mu\right)(A) \equiv 0$. This implies that Proposition 5.3 may fail without some assumption of continuity for the games.

Note for instance that the condition $d^{-} \nu(A)(A) \geq 0$ for all $A$, implies $\nu \geq 0$ (use (5.3) by setting $A=\varnothing$ ).

\section{$5.1 \quad$ Differentiation rules}

The linearity rule (3.1) entails that the addition formulas hold with no restriction. Hence, $d^{+}\left(a_{1} \nu+a_{2} \lambda\right)=a_{1} d^{+} \nu+a_{2} d^{+} \lambda$ and so on. Product formulas require much more elaboration. Before formulating them it is useful to remark a few facts.

i) The familiar multiplicative rule needs some restrictions based on the non-atomiqueness, as argued in [10]. To realize this, calculate the derivative of the product $\nu \lambda$ of two finite games. From (4.1),

$$
\begin{aligned}
D^{+}(\nu \lambda)(A)\left(\omega_{i}\right)= & \nu(A) D^{+} \lambda(A)\left(\omega_{i}\right)+\lambda(A) D^{+} \nu(A)\left(\omega_{i}\right) \\
& +D^{+} \lambda(A)\left(\omega_{i}\right) D^{+} \nu(A)\left(\omega_{i}\right)
\end{aligned}
$$

for every atom $\omega_{i} \notin A$. Consequently, the usual multiplicative rule fails. A generalization of rule (5.4), taking into account atoms, will be the object of Theorem 5.2. 
ii) The product of two differentiable set functions may be non-differentiable. Consider games over $\mathbb{N}$ equipped with the algebra $\mathcal{A}_{0}$ of finite or cofinite subsets of $\mathbb{N}$. It is easy to check that a finite charge $m$ on $\mathcal{A}_{0}$ is countably additive iff $\sum_{i=1}^{\infty} m(i)<\infty$ and $m(\mathbb{N})=\sum_{i=1}^{\infty} m(i)$. Notice that $m$ is not bounded if the series $\sum_{i=1}^{\infty} m(i)$ does not converge absolutely. Define the game $\nu=m^{2}$, with $m$ countably additive measures. Clearly $\nu$ is continuous. In view of (ii) Proposition 3.3 the derivative $D^{+} \nu(\varnothing)$ would be a countably additive measure. Set $m(i)=(-1)^{i} i^{-1 / 2}$. This would imply $D^{+} \nu(\varnothing)(i)=$ $i^{-1}$, but this measure is not finite and thus $\nu$ is not differentiable. Note incidentally that it is easy to prove that on the algebra $\mathcal{A}_{0}$ derivability implies differentiability. Hence, not even the derivative $d^{+} \nu(\varnothing)$ exists for the game $m^{2}$.

The next result enucleates the conditions that guarantee the validity of the familiar product differentiation rule.

Proposition 5.4. Assume that $\nu$ and $\lambda$ be bounded set functions and $D^{+} \nu(A)$ and $D^{+} \lambda(A)$ exist. If at least one of the two derivatives is strongly continuous, then the product $\nu \lambda$ is outer differentiable at $A$ and

$$
D^{+}(\nu \lambda)(A)=\nu(A) D^{+} \lambda(A)+\lambda(A) D^{+} \nu(A) .
$$

A similar result is valid for inner derivatives.

Though observation (ii) above shows that the set of the differentiable games is not necessarily closed by multiplication, we state that this is true under some additional regularity on the space $(\Omega, \Sigma)$. More interestingly, the derivative of the product can be computed. In the formulas below the measure $\left[D^{+} \nu(A) \otimes D^{+} \lambda(A)\right]_{\Delta}$ is the diagonal measure mentioned in the preliminary section.

Theorem 5.2. If $(\Omega, \Sigma)$ is a standard Borel space, the class of games in $\mathcal{C}$ which are outer differentiable is an algebra and

$$
\begin{aligned}
D^{+} \lambda \nu(A)= & \lambda(A) D^{+} \nu(A)+\nu(A) D^{+} \lambda(A) \\
& +\left[D^{+} \nu(A) \otimes D^{+} \lambda(A)\right]_{\Delta} .
\end{aligned}
$$

A similar result holds for the inner differentiable games and

$$
\begin{aligned}
D^{-} \lambda \nu(A)= & \lambda(A) D^{-} \nu(A)+\nu(A) D^{-} \lambda(A) \\
& -\left[D^{-} \nu(A) \otimes D^{-} \lambda(A)\right]_{\Delta} .
\end{aligned}
$$

The diagonal measures displayed in this theorem exhibit a very special structure, as established in the next Proposition. The condition for the standard product rule to hold descends easily from it. 
Proposition 5.5. The measure $\left[D^{+} \nu(A) \otimes D^{+} \lambda(A)\right]_{\Delta}$ is purely atomic. If $S_{1}$ and $S_{2}$ are the sets of the atoms of $D^{+} \nu(A)$ and $D^{+} \lambda(A)$ respectively, then

$$
\left[D^{+} \nu(A) \otimes D^{+} \lambda(A)\right]_{\Delta}(H)=\sum_{s \in S_{1} \cap S_{2} \cap H} D^{+} \nu(A)(s) D^{+} \lambda(A)(s) .
$$

Accordingly, the differentiation rule (5.5) holds iff $D^{+} \nu(A)$ and $D^{+} \lambda(A)$ have no common atoms.

As pol $(\Sigma) \subseteq p M_{\infty}(\Sigma) \subseteq p C_{\infty}(\Sigma)$, Proposition 4.2 ensures that any polynomial of measures is differentiable. Under standardness hypothesis the derivatives can be calculated by the product rule of Theorem 5.2.

Corollary 5.1. On the standard Borel space all the polynomials $\nu=q\left(\lambda_{1}, \lambda_{2}, \ldots, \lambda_{n}\right)$ of measures $\lambda_{i} \in c a$ are differentiable. The differential $D \nu(A)$ is a linear combination of the measures

$$
\lambda_{i},\left(\lambda_{i} \otimes \lambda_{j}\right)_{\Delta},\left(\lambda_{i} \otimes \lambda_{j} \otimes \lambda_{k}\right)_{\Delta}, \ldots \ldots,\left(\lambda_{i_{1}} \otimes \lambda_{i_{2}} \otimes \ldots . . \otimes \lambda_{i_{n}}\right)_{\Delta}
$$

where the indices may be repeated. Specifically,

$$
\begin{aligned}
D^{-} \lambda^{n}(A) & =\sum_{k=1}^{n}(-1)^{k+1}\left(\begin{array}{l}
n \\
k
\end{array}\right) \lambda^{n-k}(A) \Lambda_{k}, \\
D^{+} \lambda^{n}(A) & =\sum_{k=1}^{n}\left(\begin{array}{l}
n \\
k
\end{array}\right) \lambda^{n-k}(A) \Lambda_{k},
\end{aligned}
$$

with $\Lambda_{k}=\left(\otimes^{k} \lambda\right)_{\Delta}$ and $\Lambda_{1} \equiv \lambda$.

\section{Potentials}

We extend now to infinite games of the concept of potential introduced by Hart and Mas-Colell's [12] for finite games.

Definition 6.1. Given a game $\nu$, a potential of $\nu$ is a game $u \in \mathcal{C}(\Sigma)$ for which $d^{-} u(A)(A)=\nu(A)$ holds for all $A$. We shall use the notation $P \nu=u$.

The restriction $u \in \mathcal{C}(\Sigma)$ is essential. Any finite game has a unique potential (see [12]). The existence as well as uniqueness is not generally assured for infinite games. If $u_{1}$ and $u_{2}$ are two potentials of $\nu$, then $d^{-}\left(u_{1}-u_{2}\right)(A)(A) \equiv 0$. By Proposition 5.3, $u_{1}-u_{2}=k$ and the potential is uniquely defined up to an additive constant. This is the reason why we convene to consider only potential games $u$ in $\mathcal{C}(\Sigma)$. It guarantees the uniqueness of the normalized potential. 
Notice that in the original definition of [12] the potential is a map $\nu \rightarrow \mathcal{P} \nu$ from a class of games to real numbers. If the class is restrictable, the potential game, according to our acceptation, is then $u(S)=\mathcal{P} \nu^{S}$ for $S \in \Sigma$ (see Remark 2.8 of [12]).

Call $\operatorname{POT}(\Sigma)$ the totality of games on $\Sigma$ having potential. The next statement is simple to prove and left to the reader.

Proposition 6.1. POT $(\Sigma)$ is a linear, symmetric and restrictable class of games.

The purpose here is to study the class $P O T(\Sigma)$ and to clarify the properties of the operator $\nu \rightarrow P \nu$ having domain $\operatorname{POT}(\Sigma)$. The following property is a first consequence of Proposition 6.1.

Proposition 6.2. We have $\mathcal{N}(u)=\mathcal{N}(\nu)$ if $u=P(\nu)$. In particular, a game $\nu$ and its potential $u$ share the same carries; $u$ is a finite game iff $\nu$ a finite game; $u$ is nonatomic iff so is $\nu$.

The following concept, inspired by the ordinary calculus, is useful to calculate potentials. A game $\nu$ is called homogeneous of degree $\alpha$, provided $d^{-} \nu(A)(A)=\alpha \nu(A)$ for some scalar $\alpha \neq 0$. If $\nu \in \mathcal{C}(\Sigma)$ is homogeneous of degree $\alpha$, then its potential is $P \nu=\alpha^{-1} \nu$. Namely, $\nu$ is an eigenvector of the operator $P$.

To clarify the nature of the operator $P$, it is worth spending some more words about the potentials of finite games. If $u_{C}$ is an unanimity game, it is easy to check that $D^{-} u_{C}(A)(A)=|C| \cdot u_{C}(A)$, i.e., $u_{C}$ is homogeneous of degree $|C|$. Hence, $P u_{C}=$ $|C|^{-1} \cdot u_{C}$. This simple observation is the source of the following well-known results (see [12], [4] and [23]).

If $\nu$ is a finite game with multilinear extension $B_{\nu}(x)$, then $u=P \nu$ has multilinear extension

$$
B_{u}(x)=\int_{0}^{1} t^{-1} B_{\nu}(t x) d t,
$$

and $\nabla B_{u}(x) \cdot x=B_{\nu}(x)$ holds for all $x \in \mathbb{R}^{n}$.

We collect here together the main properties of the potentials of finite games. They descend easily from the relation (6.1) and are left to the reader.

Proposition 6.3. The potential operator $P$ over finite games preserves positivity, monotonicity and convexity. Moreover, $P \nu=\nu$ iff $\nu$ is additive. Finally, if $\nu$ is a symmetric game $\nu(A)=f(|A|)$ with $f:\{0,1, \ldots, n\} \rightarrow \mathbb{R}$ and $f(0)=0$, then $u=P \nu$ is symmetric as well and $u(A)=F(|A|)$ with

$$
F(r)=\sum_{i=1}^{r} i^{-1} f(i)
$$

for $r \in\{1, \ldots, n\}$. 
Many of this nice properties may fail for games with infinitely many players. The issue whether $P$ preserves monotonicity (i.e., $\nu_{1} \succeq \nu_{2} \Longrightarrow P \nu_{1} \succeq P \nu_{2}$ ) will play a key role in value theory discussed in the next section.

We turn to the existence of potentials for games in $p M_{\infty}$. We start with polynomial games.

Proposition 6.4. Any polynomial game $\nu \in$ pol $M$ admits a potential $P \nu \in$ polM.

To establish the existence of potentials in $p M_{\infty}$, the inner second order variation of a game is needed.

Assume that $\nu$ has inner derivative $d^{-} \nu(A)$ at all $A \in \Sigma$. It will be convenient here regarding $d^{-} \nu(A)(\cdot)$ as defined on the whole space, by identifying $d^{-} \nu(A)(\cdot)$ with $\widetilde{d}^{-} \nu(A)(\cdot)$ (see Section 3.2). Fix a coalition $H \in \Sigma$ and define the set function $\varphi_{H}^{-}(A)=$ $d^{-} \nu(A)(H \cap A)$ for all $A \in \Sigma$.

Definition 6.2. Set $d_{2}^{--} \nu(A)(H, K)=d^{-} \varphi_{H}^{-}(A)(K)$, whenever it does exist. Call the game $\nu$ regularly inner twice differentiable at $A$, if $d_{2}^{--} \nu(A)(H, K)$ exists for all $H, K \subseteq A$ and $d_{2}^{--} \nu(A)(H, K)=d_{2}^{--} \nu(A)(K, H)$.

We may also replace the operators $d^{-}$and $d_{2}^{--}$by $D^{-}$and $D_{2}^{--}$, whenever it is the case.

Any finite game is regularly inner twice differentiable. In fact one has

$$
D_{2}^{--} \nu(A)\left(s_{1}, s_{2}\right)=\nu(A)-\nu\left(A \backslash s_{1}\right)-\nu\left(A \backslash s_{2}\right)+\nu\left(A \backslash\left\{s_{1}, s_{2}\right\}\right) .
$$

Another simple example is $\nu=f \circ \mu$ with $f$ of class $C^{2}$ and $\mu$ nonatomic. We have

$$
D_{2}^{--} \nu(A)(H, K)=\mu(H) \cdot \nabla^{2} f(\mu(A)) \mu(K)+\nabla f(\mu(A)) \cdot \mu(H \cap K)
$$

for all $H, K \subseteq A$, and $\nu$ is regularly twice differentiable.

The role played by Definition 6.2 appears in the next Lemma.

Lemma 6.1. The potential $u=P \nu$ is monotone whenever $\nu$ is monotone, if the following two conditions hold:

i) $u$ is regularly inner twice differentiable for all $A \in \Sigma$,

ii) the set function $A \rightarrow d^{-} u(A)(H \cap A)$ is measure-continuous for all $H$.

A consequence of Lemma 6.1 is the following relevant result on the existence of potentials in $p M_{\infty}$.

Theorem 6.1. Any game $\nu \in p M_{\infty}$ admits a potential in $p M_{\infty}$. The operator $P$ preserves monotonicity and $\|P \nu\|_{\infty} \leq\|\nu\|_{\infty}$. 


\section{Value}

In this section we study the value of games according to Hart and Mas-Colell's potential theory.

If $\nu$ is a finite game and $u=P \nu$ is its potential, uniquely defined through $D^{-} u(A)(A)=$ $\nu(A)$, the Shapley value $S h \nu$ of the game $\nu$ is then the refinement derivative at $\Omega$ of the potential, that is, $S h \nu=D^{-} u(\Omega)$.

Although this relation is extensively discussed in [12], it may be here quickly checked. By (4.2) and (6.1),

$$
D^{-} u(\Omega)(S)=\sum_{s \in S} \frac{\partial B_{u}\left(1_{\Omega}\right)}{\partial x_{s}}=\sum_{s \in S} \int_{0}^{1} \frac{\partial B_{\nu}\left(t 1_{\Omega}\right)}{\partial x_{s}} d t
$$

which is the desired result in view of Owen's diagonal formula [28].

Motivated by this result, we study the operator $\psi: \operatorname{POT}(\Sigma) \rightarrow c a(\Sigma)$ defined as $\psi \nu=d^{-} u(\Omega)$ for $\nu \in \operatorname{POT}(\Sigma)$ and $u=P \nu$.

The following properties of $\psi$ are an immediate consequence of its definition and Proposition 6.2. We convene here that the underlying players' space is the standard Borel space.

Proposition 7.1. The operator $\psi:$ POT $\rightarrow$ ca satisfies the following properties:

i) $\psi$ is linear, symmetric and efficient,

ii) $\psi$ satisfies the projection axiom, i.e., $\psi \mu=\mu$ for all $\mu \in c a$,

iii) $\psi$ satisfies the null player axiom, i.e., $(\psi \nu)(N)=0$ for any $\nu$-null $N$,

iv) $\psi \nu \equiv S h \nu$, if $\nu$ has a finite carrier,

v) $\psi$ is consistent (see Section 8).

Despite of these many desirable properties, $\psi$ is not a value since the positivity axiom is not generally fulfilled (in fact, we are not able to prove that $\psi \nu \geq 0$, whenever $\nu$ is monotone). The space POT is likely to be too large and must be restrained. We deliver here two alternative subspaces of POT on which the operator $\psi$ is positive.

In view of Theorem 6.1, a first important result is obtained by restricting the operator to the space $p M_{\infty}$ that is clearly a linear, symmetric and restrictable subspace of POT.

Theorem 7.1. The operator $\psi$ is a value over $p M_{\infty} \subset$ POT and satisfies the Milnor axiom (see [22])

$$
\nu_{*} \leq \psi \nu \leq \nu^{*}
$$

$\psi$ is $\|\cdot\|_{\infty}$-continuous with $\|\psi\| \leq 1$. The solution $\psi$ coincides with the asymptotic value and its restriction to $p N A_{\infty}$ is the unique Aumann-Shapley value. 
Recall that the asymptotic value exists for these games. Actually, $A S Y M P T \supset b v^{\prime} M \supset p M_{\infty}$, see Neyman [25], [26]. The uniqueness of the value on $p N A_{\infty}$ is extensively discussed in [22] and [13]. Note further that, unlike $p N A_{\infty}$, the value on $p M_{\infty}$ is not unique. Hart [11] proved that in mixed games there are infinitely many values, corresponding to various ways in which the atomic players can be imbedded in the ocean of the negligible players.

We can in principle calculate the value of games in $p M_{\infty}$, at least this is not difficult for polynomial games, thanks to the product rule of Theorem 5.2. For instance, if $\nu_{2}=\lambda_{1} \lambda_{2}$ with $\lambda_{1}, \lambda_{2} \in c a$,

$$
\begin{aligned}
P \nu_{2} & =2^{-1}\left[\lambda_{1} \lambda_{2}+\left(\lambda_{1} \otimes \lambda_{2}\right)_{\Delta}\right] \\
\psi \nu_{2} & =2^{-1}\left[\lambda_{1}(\Omega) \lambda_{2}+\lambda_{2}(\Omega) \lambda_{1}\right],
\end{aligned}
$$

that turns out curiously to be like the nonatomic case. However, this simple formula is no longer preserved by monomials $\nu_{n}=\lambda_{1} \lambda_{2} \cdot \ldots . \lambda_{n}$. For example,

$$
\begin{aligned}
\psi \nu_{3}= & 3^{-1} \sum_{i \neq j \neq k}\left(\lambda_{i} \lambda_{j}\right)(\Omega) \lambda_{k}-6^{-1} \sum_{i \neq j \neq k} \lambda_{i}(\Omega)\left(\lambda_{j} \otimes \lambda_{k}\right)_{\Delta} \\
& +6^{-1} \sum_{i \neq j \neq k}\left(\lambda_{j} \otimes \lambda_{k}\right)_{\Delta}(\Omega) \lambda_{i} .
\end{aligned}
$$

Another simple example is the value of games $\nu=\lambda^{n} u_{T}$ with $\lambda \in n a^{1}$ and $u_{T}$ is an unanimity game, where $T$ is a finite coalition. By the product rule of Proposition 5.4 it follows that $\lambda^{n} u_{T}$ is homogeneous of degree $n+|T|$. Therefore

$$
(\psi \nu)(H)=\frac{|T|}{n+|T|} \frac{|T \cap H|}{|T|}+\frac{n}{n+|T|} \lambda(H) .
$$

In order to specify another available restriction of $P O T$, we focus on an important property exhibited by a solution concept.

Let $\nu$ be a finite game with potential $u$. Since finite games are regularly inner twice differentiable, $D_{2}^{--} u(\Omega)(i, j)=D_{2}^{--} u(\Omega)(j, i)$, according to Definition 6.2. By (6.2),

$$
D^{-} u(\Omega)(i)-D^{-} u(\Omega \backslash j)(i)=D^{-} u(\Omega)(j)-D^{-} u(\Omega \backslash i)(j),
$$

if $i \neq j$. That is, $S h_{i}(\nu)-S h_{i}\left(\nu^{\Omega \backslash j}\right)=S h_{j}(\nu)-S h_{j}\left(\nu^{\Omega \backslash i}\right)$ which is the well-known Myerson's balanced contribution property [24] (see also [12]). The extension of this property to large games is straightforward by means of refinement limits.

If $\phi$ is a solution concept defined over a restrictable class of games, $\phi$ is said to satisfy the balanced contribution axiom, provided

$$
\lim _{\pi \in \Pi(K)} \sum_{K_{j} \in \pi} \phi\left(\nu^{S}\right)(H)-\phi\left(\nu^{S \backslash K_{j}}\right)(H)=\lim _{\pi \in \Pi(H)} \sum_{H_{i} \in \pi} \phi\left(\nu^{S}\right)(K)-\phi\left(\nu^{S \backslash H_{i}}\right)(K)
$$


holds for all $S \in \Sigma$ and all coalitions $H, K \subseteq S$ such that $H \cap K=\varnothing$. When $\phi$ coincides with operator $\psi$, this condition clearly amounts to saying that the potential is regularly twice inner differentiable.

In view of Lemma 6.1 we are thus able to enucleate another subspace of POT.

Denote $\mathrm{POT}_{2} \subset P O T$ the totality of games whose potentials satisfy the conditions (i)-(ii) of Lemma 6.1. Observe that condition (ii) entails that $P O T_{2} \subset \mathcal{C}$.

Theorem 7.2. $P O T_{2}$ is a linear, symmetric and restrictable space. The restriction to $P O T_{2}$ of the operator $\psi$ is a value that satisfies the balanced contribution axiom.

The two subspaces $p M_{\infty}$ and $P O T_{2}$ are not comparable. There are games in $p M_{\infty}$ which are not twice differentiable and consequently the balanced contribution axiom is not valid on $p M_{\infty}$. On the other hand, $P O T_{2}$ is not included into $p M_{\infty}$. Consider for instance the games of type $\nu(A)=\lambda(A \times A)$ described in Lemma 8.1 of Section 8 . They lie in $\mathcal{L}$ but not necessarily in $p M_{\infty}$. It is easy to see that these games belong to $P O T_{2}$. Note incidentally that $P \nu=2^{-1}\left(\nu+\lambda_{d}\right)$ and $\psi \nu=\lambda_{m}$ holds for such games, where $\lambda_{d}$ and $\lambda_{m}$ are measures defined therein.

\section{Proofs}

Proposition 3.1. i) From $-|\mu(E)-m(E)| \leq \mu(E)-m(E) \leq|\mu(E)-m(E)|$, it follows that $|\mu-m| \rightarrow_{\pi} 0 \Longrightarrow \mu-m \rightarrow_{\pi} 0 \Longleftrightarrow \mu \rightarrow_{\pi} m$. By the uniqueness of the limit, $m=\mu_{\#}$. Note further that if $n_{\pi} \equiv n_{\pi}^{|\mu-m|}$ is the measures associated with the set function $|\mu-m|$, the $n_{\pi}$ are clearly positive. Given $E$, we have $0 \leq n_{\pi}(E) \leq n_{\pi}(\Omega)$ for all $\pi \succeq\left\{E, E^{c}\right\}$. Hence, $n_{\pi}(E) \rightarrow 0$ whenever $n_{\pi}(\Omega) \rightarrow 0$ that implies the second statement.

ii) Assume that $\mu \rightarrow_{\pi} \mu_{\#}$ uniformly. Fix $E \in \mathcal{A}$ and $\varepsilon>0$. There is $\pi^{0} \in \Pi(\Omega)$ such that if $\pi \succeq \pi_{E}^{0},\left|\sum_{E_{i} \in \pi} \mu\left(E_{i}\right)-\mu_{\#}\left(E_{i}\right)\right|<\varepsilon$. Set $\pi^{+}=\left\{E_{i} \in \pi: \mu\left(E_{i}\right) \geq \mu_{\#}\left(E_{i}\right)\right\}$ and $\pi^{-}=\left\{E_{i} \in \pi: \mu\left(E_{i}\right)<\mu_{\#}\left(E_{i}\right)\right\}$. Clearly, $\pi^{+}$is a partition of a subset $B \subseteq E$ and $\pi^{-}$ is a partition of $E \backslash B$ and $\pi^{+} \succeq \pi_{B}^{0}, \pi^{-} \succeq \pi_{E \backslash B}^{0}$. Consequently, as $\mu \rightarrow_{\pi} \mu_{\#}$ holds uniformly, we have

$$
\begin{aligned}
\sum_{E_{i} \in \pi^{+}}\left|\mu\left(E_{i}\right)-\mu_{\#}\left(E_{i}\right)\right| & =\sum_{E_{i} \in \pi^{+}} \mu\left(E_{i}\right)-\mu_{\#}\left(E_{i}\right)<\varepsilon \\
\sum_{E_{i} \in \pi^{-}}\left|\mu\left(E_{i}\right)-\mu_{\#}\left(E_{i}\right)\right| & =-\sum_{E_{i} \in \pi^{-}} \mu\left(E_{i}\right)-\mu_{\#}\left(E_{i}\right)<\varepsilon .
\end{aligned}
$$

Therefore, $\sum_{E_{i} \in \pi}\left|\mu\left(E_{i}\right)-\mu_{\#}\left(E_{i}\right)\right|<2 \varepsilon$ and $\left|\mu-\mu_{\#}\right| \rightarrow_{\pi} 0$.

Conversely, suppose $\mu \rightarrow_{\pi} \mu_{\#}$ absolutely. Let $m_{\pi}$ be the measures associated with the set function $\left|\mu-\mu_{\#}\right|$. Given $\varepsilon>0$, there is $\pi^{0} \in \Pi(\Omega)$ such that $m_{\pi}(\Omega)<\varepsilon$ for all 
$\pi \succeq \pi^{0}$. Consequently, $m_{\pi}(E)<\varepsilon$ for all $\pi \succeq \pi^{0} \vee\left\{E, E^{c}\right\}$ and all $E$. This means that $\left|\mu-\mu_{\#}\right| \rightarrow_{\pi} 0$ holds uniformly. Consequently, $\mu \rightarrow_{\pi} \mu_{\#}$ uniformly.

Proposition 3.2. We treat only the set function $\varphi^{3}(X)=\nu(A \cup X)-\nu(A \backslash X) \equiv$ $\Delta \nu(A)(X)$. The other cases are similar. Observe first that $\Delta \nu(A)(H)=\Delta^{+} \nu(A)(H)$, if $H \subseteq A^{c}$, and $\Delta \nu(A)(K)=\Delta^{-} \nu(A)(K)$, if $K \subseteq A$. Thus, if $\varphi^{3} \rightarrow_{\pi} \varphi_{\#}^{3}$, then $\Delta^{+} \nu(A) \rightarrow_{\pi}\left(\varphi_{\#}^{3}\right)_{A^{c}}=d^{+} \nu(A)$. Likewise, $\Delta^{-} \nu(A) \rightarrow_{\pi}\left(\varphi_{\#}^{3}\right)_{A}=d^{-} \nu(A)$.

Conversely, assume that $d^{+} \nu(A)$ and $d^{-} \nu(A)$ exist. Consider the two set functions $\varphi^{1}(X)=\nu(A \cup X)-\nu(A)$ and $\varphi^{2}(X)=\nu(A)-\nu(A \backslash X)$, both defined on $\mathcal{A}$. It is easy to check that $\varphi_{\#}^{1}$ and $\varphi_{\#}^{2}$ exist and $\left(\varphi_{\#}^{1}\right)_{A}=0,\left(\varphi_{\#}^{1}\right)_{A^{c}}=d^{+} \nu(A)$. Likewise, $\left(\varphi_{\#}^{2}\right)_{A}=d^{-} \nu(A),\left(\varphi_{\#}^{2}\right)_{A^{c}}=0$. Therefore, as $\Delta \nu(A)(X)=\nu(A \cup X)-\nu(A)+\nu(A)-$ $\nu(A \backslash X)=\varphi^{1}(X)+\varphi^{2}(X)$, we deduce the existence of the refinement limit of $\Delta \nu(A)$.

Proposition 3.3. i) If $\nu$ is bounded, $\left|\Delta^{+} \nu(A)(H)\right| \leq M$ holds for all $A$ and $H$ and for some $M \geq 0$. By Definition 3.4 and Proposition 3.1, $\Delta^{+} \nu(A) \rightarrow_{\pi} D^{+} \nu(A)$ uniformly. Set $\varepsilon=1$. There is a partition $\pi^{0} \in \Pi\left(A^{c}\right)$ such that

$$
\left|D^{+} \nu(A)(H)-\sum_{H_{i} \in \pi_{H}^{0}} \Delta^{+} \nu(A)\left(H_{i}\right)\right|<1,
$$

holds for all $H \in \Sigma_{A^{c}}$. If $n$ is the cardinality of the partition $\pi^{0}$, we get

$$
\begin{aligned}
\left|D^{+} \nu(A)(H)\right| & \leq\left|D^{+} \nu(A)(H)-\sum_{H_{i} \in \pi_{H}^{0}} \Delta^{+} \nu(A)\left(H_{i}\right)\right| \\
+\left|\sum_{H_{i} \in \pi_{H}^{0}} \Delta^{+} \nu(A)\left(H_{i}\right)\right| & <1+n M .
\end{aligned}
$$

ii) If $\nu$ is outer continuous at $A$, then the game $\Delta^{+} \nu(A)(\cdot)$ is continuous at $\varnothing$. Let $H_{n} \downarrow \varnothing$, with $H_{n} \in \mathcal{A}_{A^{c}}$, and fix $\varepsilon>0$. There is a partition $\pi^{0}=\left\{K_{i}\right\}_{i=1}^{r} \in \Pi\left(A^{c}\right)$ whereby $\sum_{B_{i} \in \pi_{H n}^{0}}\left|\Delta^{+} \nu(A)\left(B_{i}\right)-D^{+} \nu(A)\left(B_{i}\right)\right| \leq \varepsilon$ for all $n$. On the other hand, $B_{i}=H_{n} \cap K_{i}$. Hence,

$$
\sum_{i=1}^{r}\left|\Delta^{+} \nu(A)\left(H_{n} \cap K_{i}\right)-D^{+} \nu(A)\left(H_{n} \cap K_{i}\right)\right| \leq \varepsilon
$$


is true for all $n$. It follows

$$
\begin{aligned}
\left|D^{+} \nu(A)\left(H_{n}\right)\right| & =\left|\sum_{i=1}^{r} D^{+} \nu(A)\left(H_{n} \cap K_{i}\right)\right| \leq \\
\sum_{i=1}^{r}\left|D^{+} \nu(A)\left(H_{n} \cap K_{i}\right)\right| & \leq \sum_{i=1}^{r}\left|D^{+} \nu(A)\left(H_{n} \cap K_{i}\right)-\Delta^{+} \nu(A)\left(H_{n} \cap K_{i}\right)\right| \\
+\sum_{i=1}^{r}\left|\Delta^{+} \nu(A)\left(H_{n} \cap K_{i}\right)\right| & \leq \varepsilon+\sum_{i=1}^{r}\left|\Delta^{+} \nu(A)\left(H_{n} \cap K_{i}\right)\right| .
\end{aligned}
$$

Since $H_{n} \cap K_{i} \downarrow \varnothing$ for all $i$, we obtain $\limsup _{n \rightarrow \infty}\left|D^{+} \nu(A)\left(H_{n}\right)\right| \leq \varepsilon$. As $\varepsilon$ is arbitrary, $D^{+} \nu(A)\left(H_{n}\right) \rightarrow 0$. This implies that $D^{+} \nu(A)(\cdot)$ is countably additive.

(iii) Set $\varphi_{A}(X)=\nu(A \cup X)-\nu(A)$ and $\varphi_{B}(X)=\nu(B \cup X)-\nu(B)$, for all $X \in \mathcal{A}$. From the relation $B=[A \backslash(A \backslash B)] \cup(B \backslash A)$ it follows

$$
\begin{aligned}
\varphi_{B}(X) & =\nu(A \backslash(A \backslash B) \cup(B \backslash A) \cup X) \\
-\nu(A \backslash(A \backslash B) \cup(B \backslash A)) & =\varphi_{A}(X) .
\end{aligned}
$$

Proposition 3.2 provides the assertion.

iv) Let $\varphi(X)=\nu(A \cup X)-\nu(A)$ for all $X \in \mathcal{A}$. It is easy to check that $\varphi \ll \nu$. It suffices to prove that $\varphi_{\#} \ll \varphi$. In that, $\nu \gg \varphi \gg \varphi_{\#}=d^{+} \nu(A)$. On the other hand, if $N \in \mathcal{N}(\varphi)$, it holds $\varphi_{\#}(E \cup N)=\varphi_{\#}(E \cup(N \backslash E))=\varphi_{\#}(E)+\varphi_{\#}(N \backslash E)$. The result follows if $\varphi_{\#}(N)=0$ whenever $N \in \mathcal{N}(\varphi)$ but this is evident.

v) This is equivalent to the fact that if $\nu$ has an atom then $\nu_{\#}=d^{+} \nu(\varnothing)$ has an atom as well. Let $A$ be an $\nu$-atom. Since for every $B \subseteq A$, either $B$ or $A \backslash B$ is $\nu$-null, by (iv) either $B$ or $A \backslash B$ is $\nu_{\#}$-null. It follows that $A$ is a $\nu_{\#}$-atom, provided $\nu_{\#}(A) \neq 0$. On the other hand if one considers a partition $\pi$ of $A$ and the relative sum $\sum_{A_{i} \in \pi} \nu\left(A_{i}\right)$ at least one $A_{i}$, say $A_{1}$ is non-null, otherwise $\nu(A)$ would vanish. But then $A \backslash A_{1}$ is $\nu$-null. Consequently, all the elements $A_{i}$ with $i>1$ are $\nu$-null. Hence, $\sum_{A_{i} \in \pi} \nu\left(A_{i}\right)=\nu(A)$ for all the partitions and, consequently, $\nu_{\#}(A)=\nu(A) \neq 0$.

Theorem 4.1. A preliminary observation is that the outer increment $\Delta^{+} \nu(A)(\cdot)$ is superadditive when $\nu$ is convex. Therefore, if $\pi_{1} \succeq \pi$, with $\pi_{1}, \pi \in \Pi(H)$,

$$
\sum_{H_{i} \in \pi} \nu\left(A \cup H_{i}\right)-\nu(A) \geq \sum_{K_{j} \in \pi_{1}} \nu\left(A \cup K_{j}\right)-\nu(A) .
$$

By monotone convergence criterion,

$$
d^{+} \nu(A)(H)=\inf _{\pi \in \Pi(H)} \sum_{H_{i} \in \pi} \nu\left(A \cup H_{i}\right)-\nu(A),
$$

provided the infimum is finite for all $H \in \mathcal{A}_{A^{c}}$. On the other hand, if $m \in \partial \nu(\varnothing) \neq \varnothing$, as $\nu$ is superadditive, $\nu\left(A \cup H_{i}\right)-\nu(A) \geq \nu\left(H_{i}\right) \geq m\left(H_{i}\right)$. We infer that $d^{+} \nu(A)(H) \geq$ 
$m(H)$ and $d^{+} \nu(A)$ exists for all $A$. Note that $d^{+} \nu(A)(H) \leq \nu(A \cup H)-\nu(A)$ that, in turn, implies $d^{+} \nu(A) \in \partial^{+} \nu(A)$. Clearly the inverse implication is obvious: if $d^{-} \nu(\varnothing)$ exists then $d^{-} \nu(\varnothing) \in \partial \nu(\varnothing)$ and thus $\partial \nu(\varnothing)$ is nonempty.

Observe next that $\nu\left(A \cup H_{i}\right)-\nu(A)-d^{+} \nu(A)\left(H_{i}\right) \geq 0$. Thus,

$$
\left|\nu\left(A \cup H_{i}\right)-\nu(A)-d^{+} \nu(A)\left(H_{i}\right)\right|=\nu\left(A \cup H_{i}\right)-\nu(A)-d^{+} \nu(A)\left(H_{i}\right) .
$$

Hence, $\left|\Delta^{+} \nu(A)-d^{+} \nu(A)\right| \rightarrow_{\pi} 0$ and $D^{+} \nu(A)=d^{+} \nu(A)$.

To conclude, let $m \in \partial^{+} \nu(A)$. Clearly, $\nu\left(A \cup H_{i}\right)-\nu(A) \geq m\left(H_{i}\right)$. Eq. (8.1) implies that $d^{+} \nu(A)(H) \geq m(H)$ for all $H \in \mathcal{A}_{A^{c}}$. Therefore (4.3) holds.

The assertion about inner differentials are obtained by duality. It suffices to observe that $\partial^{+} \nu(A)=\bar{\partial}^{-} \bar{\nu}\left(A^{c}\right)$, where the dual operator $\bar{\partial}$ denotes superdifferentials in place of subdifferentials, and $d^{-} \nu(\Omega)=d^{+} \bar{\nu}(\varnothing)$. The proof goes through as before.

Next we prove statement (iii). Assume first that $\nu \geq 0$. By superadditivity, $\nu$ is monotone. Fixed $A$, for any $B$ we have

$$
\begin{aligned}
\nu(B) & \geq \nu(A \cap B) \geq \nu(A)+D^{-} \nu(A)(A \cap B)-D^{-} \nu(A)(A) \\
& =\nu(A)+\widetilde{D}^{-} \nu(A)(B)-\widetilde{D}^{-} \nu(A)(A),
\end{aligned}
$$

which implies $\widetilde{D}^{-} \nu(A) \in \partial \nu(A)$. If $\nu$ is not monotone and $m \in \partial \nu(\varnothing)$, then $\nu_{1}=\nu-$ $m \geq 0$. By the previous argument, $\widetilde{D}^{-} \nu_{1}(A)=\widetilde{D}^{-} \nu(A)-m^{A} \in \partial \nu_{1}(A)=\partial \nu(A)-m$. It follows, $D^{-} \nu(A) \oplus m_{A^{c}} \in \partial \nu(A)$ and this shows that $\nu$ is subdifferentiable at any $A$. In particular, we can take $m=D^{+} \nu(\varnothing)$.

iv) We know that an equivalent condition for convexity is that $\nu(A \cup H)-\nu(A) \leq$ $\nu(B \cup H)-\nu(B)$ for $A \subseteq B$ and $B \cap H=\varnothing$. It follows that

$$
\sum_{H_{i} \in \pi} \nu\left(A \cup H_{i}\right)-\nu(A) \leq \sum_{H_{i} \in \pi} \nu\left(B \cup H_{i}\right)-\nu(B)
$$

holds for any partition $\pi$ of $H$. Taking the limit, $D^{+} \nu(A)(H) \leq D^{+} \nu(B)(H)$ for each $H \in \mathcal{A}_{B^{c}}$. In the same way we obtain $D^{-} \nu(A)(K) \leq D^{-} \nu(B)(K)$ for all $K \in \mathcal{A}_{A}$. Let now $H \subseteq B \backslash A$. From point (i), we have $\nu(A \cup H)-\nu(A) \geq D^{+} \nu(A)(H)$ and $\nu(A)-\nu(A \cup H) \geq-D^{-} \nu(A \cup H)(H)$. Summing up, we get $D^{-} \nu(A \cup H)(H) \geq$ $D^{+} \nu(A)(H)$. Consequently, $D^{-} \nu(B)(H) \geq D^{-} \nu(A \cup H)(H) \geq D^{+} \nu(A)(H)$. Putting together these results, we obtains $D \nu(A) \leq D \nu(B)$, provided $A \subseteq B$.

Proposition 4.1. One implication is obvious. If $\nu$ is $m$-Lipschitz, then $-m \in$ $\partial \nu(\varnothing)$ and $m \in \partial \nu(\Omega)$ with $m \in c a(\Sigma)$. Conversely, assume that $\nu$ is convex and $\partial \nu(\varnothing) \neq \varnothing$ and $\partial \nu(\Omega) \neq \varnothing$. Let $A \subseteq B$. By Theorem 4.1, $\nu$ is differentiable and $D^{+} \nu(A) \in \partial^{+} \nu(A)$. Therefore, $-D^{+} \nu(A)(B \backslash A) \geq \nu(A)-\nu(B)$.

By (iv) of Theorem $4.1,-D^{+} \nu(\varnothing)(B \backslash A) \geq-D^{+} \nu(A)(B \backslash A)$. Therefore $-D^{+} \nu(\varnothing)(B \backslash A) \geq$ $\nu(A)-\nu(B)$. 
Similarly, $D^{-} \nu(\Omega)(B \backslash A) \geq D^{-} \nu(B)(B \backslash A) \geq \nu(B)-\nu(A)$.

Note that by (ii) of Proposition $3.3, D^{+} \nu(\varnothing)$ and $D^{-} \nu(\Omega)$ belong to $c a(\Sigma)$ as $\nu$ is continuous. Setting $m=D^{-} \nu(\Omega) \vee\left[-D^{+} \nu(\varnothing)\right] \vee 0 \in c a(\Sigma)$, we get $|\nu(B)-\nu(A)| \leq$ $m(B)-m(A)$ and $\nu$ is Lipschitz.

Theorem 4.2. If $\nu$ is Lipschitz it is easy to see that

$$
-\|\nu\|_{\infty} \leq \underline{d}^{+} \nu(A)(H) \leq \bar{d}^{+} \nu(A)(H) \leq\|\nu\|_{\infty}
$$

holds for all $A$ and $H \in \Sigma_{A^{c}}$.

Now, if $\nu_{n} \rightarrow u$ in $\mathcal{L}(\Sigma)$, the sequence $\nu_{n}$ is Cauchy. Applying (8.2) to $\nu_{n}-\nu_{m}$, we get $\left|d^{+} \nu_{n}(A)(H)-d^{+} \nu_{m}(A)(H)\right| \leq\left\|\nu_{n}-\nu_{m}\right\|_{\infty}$. It follows $d^{+} \nu_{n}(A)(H) \rightarrow \Phi(A, H)$ for all $A$ and $H \in \Sigma_{A^{c}}$ and the convergence is uniformly on $\Sigma_{A^{c}}$. On the other hand, by the decomposition $u=\left(u-\nu_{n}\right)+\nu_{n}$, we have

$$
\begin{aligned}
& \underline{d}^{+} u(A)=\underline{d}^{+}\left(u-\nu_{n}\right)(A)+d^{+} \nu_{n}(A) \\
& \bar{d}^{+} u(A)=\bar{d}^{+}\left(u-\nu_{n}\right)(A)+d^{+} \nu_{n}(A) .
\end{aligned}
$$

From (8.2),

$$
\begin{aligned}
\left|\underline{d}^{+} u(A)(H)-d^{+} \nu_{n}(A)(H)\right| & \leq\left\|u-\nu_{n}\right\|_{\infty} \\
\left|\bar{d}^{+} u(A)(H)-d^{+} \nu_{n}(A)(H)\right| & \leq\left\|u-\nu_{n}\right\|_{\infty} .
\end{aligned}
$$

Consequently, $\underline{d}^{+} u(A)(H)=\bar{d}^{+} u(A)(H)=\Phi(A, H)=\lim _{n} d^{+} \nu_{n}(A)(H)$ which proves $u$ is outer differentiable as well as the limit property.

Suppose now that $d^{+} \nu_{n}(A)=D^{+} \nu_{n}(A)$. By the decomposition $u=\left(u-\nu_{n}\right)+\nu_{n}$, it follows

$$
\begin{aligned}
& \sum_{i}\left|u\left(A \cup H_{i}\right)-u(A)-d^{+} u(A)\left(H_{i}\right)\right| \\
\leq & 2\left\|u-\nu_{n}\right\|+\sum_{i}\left|\nu_{n}\left(A \cup H_{i}\right)-\nu_{n}(A)-d^{+} \nu_{n}(A)\left(H_{i}\right)\right| .
\end{aligned}
$$

From which it is easy to prove that $d^{+} u(A)=D^{+} u(A)$. The result for the inner derivatives is analogous or, more simply, observe that the conjugate game $\bar{\nu}$ is Lipschitz and $\|\nu\|_{\infty}=\|\bar{\nu}\|_{\infty}$.

Proposition 4.2. Observe first that every Lipschitz convex game $\nu$ is the difference $\nu=\nu^{+}-\nu^{-}$of two Lipschitz monotone convex games. To see this, note that $\partial \nu(\varnothing) \cap$ $c a(\Sigma) \neq \varnothing$ follows from Proposition 4.1. If $m \in \partial \nu(\varnothing) \cap c a(\Sigma)$, then $\nu \geq m \geq-m^{-}$. Hence, $\nu=\nu^{+}-\nu^{-}$with $\nu^{+}=\nu+m^{-}$and $\nu^{-}=m^{-}$. 
Let $p\left(\nu_{1}, \nu_{2}, \ldots, \nu_{n}\right)$ be a polynomial of Lipschitz convex games $\nu_{i}$. By the decomposition $\nu_{i}=\nu_{i}^{+}-\nu_{i}^{-}$, we get $p\left(\nu_{1}, \nu_{2}, \ldots, \nu_{n}\right)=q\left(\nu_{1}^{+}, . ., \nu_{n}^{+}, \nu_{1}^{-}, . ., \nu_{n}^{-}\right)$where $q$ is another polynomial. Now we can obtain the decomposition $q=q^{+}-q^{-}$where the coefficients of the two polynomials $q^{+}$and $q^{-}$are positive. It is well-known that the product of two positive convex games is convex, consequently, $q^{+}\left(\nu_{1}^{+}, . ., \nu_{n}^{+}, \nu_{1}^{-}, . ., \nu_{n}^{-}\right)$ and $q^{-}\left(\nu_{1}^{+}, . ., \nu_{n}^{+}, \nu_{1}^{-}, . ., \nu_{n}^{-}\right)$are convex and Lipschitz. By Theorem 4.1 it follows that $p\left(\nu_{1}, \nu_{2}, \ldots, \nu_{n}\right)$ is differentiable. Theorem 4.2 implies that every member of $p C_{\infty}(\Sigma)$ is differentiable.

Proposition 4.3. The Lipschitz continuity at $\bar{x}$ ensures that $f$ has the Frechet property with its directional derivative $f^{\prime}(\bar{x} ; h)$, that is

$$
\lim _{\|h\| \rightarrow 0, h \geq 0}\|h\|^{-1}\left|f(\bar{x}+h)-f(\bar{x})-\nabla^{+} f(\bar{x}) \cdot h\right|=0 .
$$

See for instance [14, Th. 3.35] for a proof. This implies that for any $\varepsilon>0$, there is a $\delta>0$ such that $\left|f(\bar{x}+h)-f(\bar{x})-\nabla^{+} f(\bar{x}) \cdot h\right| \leq \varepsilon\|h\|$, provided $h \geq 0$ and $\|h\| \leq \delta$. As $\mu$ is strongly continuous, a partition $\pi^{0} \in \Pi\left(A^{c}\right)$ exists such that if $\pi \succeq \pi^{0}$ then $\left\|\mu\left(H_{i}\right)\right\| \leq \delta$ for all $H_{i} \in \pi$ and thus

$$
\begin{aligned}
\left|f\left(\mu(A)+\mu\left(H_{i}\right)\right)-f(\mu(A))-\nabla^{+} f(\mu(A)) \cdot \mu\left(H_{i}\right)\right| & \leq \varepsilon\left\|\mu\left(H_{i}\right)\right\| \\
\left|\nu\left(A \cup H_{i}\right)-\nu(A)-\nabla^{+} f(\mu(A)) \cdot \mu\left(H_{i}\right)\right| & \leq \varepsilon\left\|\mu\left(H_{i}\right)\right\| .
\end{aligned}
$$

Summing up,

$$
\sum_{H_{i} \in \pi}\left|\nu\left(A \cup H_{i}\right)-\nu(A)-\nabla^{+} f(\mu(A)) \cdot \mu\left(H_{i}\right)\right| \leq \varepsilon|\mu|\left(A^{c}\right) .
$$

By virtue of (i) of Proposition 3.1, $D^{+} \nu(A)=\nabla^{+} f(\mu(A)) \cdot \mu_{A^{c}}$. The remaining result is easily obtained by duality. For, $D^{-} \nu(A)=D^{+} \bar{\nu}\left(A^{c}\right)$ and $\bar{\nu}(E)=\bar{f}(\mu(E))$, where $\bar{f}(x)=f(\mu(\Omega))-f(\mu(\Omega)-x)$.

Proposition 4.4. Observe that if $\left\{H_{i}\right\}_{i=1}^{n}$ is a fixed partition of $A^{c}$, we can consider the convergence by refinement of $\Delta^{+} \nu(A)(\cdot)$ on each single $H_{i}$. The limit, whenever it does exist, can be seen as a "partial" derivative $d_{H_{i}}^{+} \nu(A) \in a\left(\Sigma_{H_{i}}\right)$. Obviously, $d^{+} \nu(A)$ exists iff each $d_{H_{i}}^{+} \nu(A)$ exists and further, $d^{+} \nu(A)=\oplus_{i=1}^{n} d_{H_{i}}^{+} \nu(A)$. The same argument holds for the differentials.

Since the measures $\mu_{i}$ are mutually singular, there is a partition $\left(\Omega_{i}\right)_{i=1}^{n}$ of $\Omega$ for which $\mu_{i}\left(\Omega_{j}\right)=0$ for all $i \neq j$. Consider the partition $\left\{H_{i}\right\} \in \Pi\left(A^{c}\right)$ with $H_{i}=A^{c} \cap \Omega_{i}$ and calculate the partial derivative $D_{H_{i}}^{+} \nu(A)$. If $K \subseteq H_{i}$, we have

$$
\nu(A \cup K)-\nu(A)=f\left(\mu(A)+\mu_{i}(K) e_{i}\right)-f(\mu(A)) .
$$


This is a scalar measure game and we can invoke Proposition 4.3. Note that in the one-dimensional case the local Lipschitz continuity condition in Proposition 4.3 is superfluous. We deduce that $D_{H_{i}}^{+} \nu(A)=f_{i}^{+}(\mu(A)) \mu_{i}$ on $H_{i}$. To conclude, if $K \subseteq A^{c}$, we have

$$
\begin{aligned}
D^{+} \nu(A)(K) & =\sum_{i=1}^{n} f_{i}^{+}(\mu(A)) \mu_{i}\left(H_{i} \cap K\right) \\
& =\sum_{i=1}^{n} f_{i}^{+}(\mu(A)) \mu_{i}(K)=\nabla^{+} f(\mu(A)) \cdot \mu(K)
\end{aligned}
$$

which is the desired result.

Proposition 5.1. $\mathcal{C}(\Sigma)$ is manifestly an algebra. Actually, if $m_{1}$ and $m_{2}$ are control measures of $\nu_{1}$ and $\nu_{2}$, respectively, then $m_{1}+m_{2}$ is a control measure of $\nu_{1} \pm \nu_{2}$ as well as of $\nu_{1} \nu_{2}$.

We prove that $\mathcal{C}(\Sigma)$ is closed by uniform limits. Let $\nu_{n} \in \mathcal{C}(\Sigma)$ with control measures $m_{n} \in c a^{1}(\Sigma)$. Define the control measure $m=\sum_{i=1}^{\infty} 2^{-i} m_{i}$. Assume that $\nu$ is the uniform limit of the sequence $\nu_{n}$, i.e., $\nu(A)=\lim _{n} \nu_{n}(A)$ for all $A \in \Sigma$, and where the set-wise limit is uniform over $A \in \Sigma$. If $A_{m}$ is a sequence for which $m\left(A_{m} \Delta A\right) \rightarrow 0$, clearly, $m_{n}\left(A_{m} \Delta A\right) \rightarrow 0$ for all $n$. From the relation

$$
\begin{aligned}
\left|\nu\left(A_{m}\right)-\nu(A)\right| \leq & \left|\nu\left(A_{m}\right)-\nu_{n}\left(A_{m}\right)\right| \\
& +\left|\nu_{n}\left(A_{m}\right)-\nu_{n}(A)\right|+\left|\nu_{n}(A)-\nu(A)\right|,
\end{aligned}
$$

it is immediate to infer the desired property.

(i) To prove that $\mathcal{C}(\Sigma)$ includes the continuous exact games, we make use of an important device already utilized in [20] and that will be basically utilized in Theorem 5.1 .

Given $(\Omega, \Sigma)$ and a positive measure $m$, we construct the metric space $\Sigma(m)$. We recall that $\Sigma(m)$ is obtained by identifying elements $F \sim G$ in $\Sigma$, if $m(F \triangle G)=0$. By these equivalence classes, one defines the metric $\rho(F, G)=m(F \triangle G)=\int\left|1_{F}-1_{G}\right| d m$ (see e.g. [6, III. 7]). The metric space $\Sigma(m)$ turns out to be complete, as it can be metrically embedded into a closed subset of $L^{1}(\Omega, \Sigma, m)$.

By the classical Schmeidler's result [32] (see also [18, Th. 4.2]), we can regard any continuous exact game $\nu$ as a function defined on the metric space $\Sigma(m)$, where the probability measure $m$ is assured to exist by Schmeidler's theorem. Moreover, as $\nu$ is the lower envelope of the additive functionals $\lambda \in \operatorname{core}(\nu) \subseteq c a(\Sigma, m)$, which are continuous in $\Sigma(m)$, the function $\nu: \Sigma(m) \rightarrow \mathbb{R}$ turns out to be upper semicontinuous. In fact, $\nu$ is continuous since the family $\mu \in \operatorname{core}(\nu)$ is equicontinuous on $\Sigma(m)$. It is easy to check that the lower envelope of a family of equicontinuous functions is lower 
semicontinuous. To conclude, $\nu: \Sigma(m) \rightarrow \mathbb{R}$ is continuous. Clearly the continuity in the metric space $\Sigma(m)$ is equivalent to the continuity in measure.

(ii) Since the polynomials of $\sigma$-additive measures lie in $\mathcal{C}(\Sigma)$, the uniform closure is included into $\mathcal{C}(\Sigma)$. Hence $p N A^{\prime} \subset \mathcal{C}(\Sigma)$. By [21], under standardness assumption, the class $p N A^{\prime}$ coincides with the games having na-continuous extensions.

(iii) and (iv) are trivial and the proof is omitted.

$(\mathrm{v})$ Pick as control measure any measure which has a positive mass at each point $i \in \mathbb{N}$, for instance $m(i)=2^{-i}$. Then, $m\left(A_{n} \triangle A\right) \rightarrow 0$ iff $\lim _{n} A_{n}=A$. To see this, assume that $m\left(A_{n} \triangle A\right) \rightarrow 0$. In view of point (i) that means that $1_{A_{n}} \rightarrow 1_{A}$ in $\ell^{1}(\mathbb{N}, m)$. It follows that $1_{A_{n}} \rightarrow 1_{A}$ point-wise, namely, $\lim _{n} A_{n}=A$. The converse implication is obvious, since $1_{A_{n}} \rightarrow 1_{A}$ point-wise implies the convergence in measure. Now the argument follows the same lines adopted by [7] and details are omitted.

Theorem 5.1. In view of point (i) in the proof of Proposition 5.1, we regard the set function $\nu$ as an upper semicontinuous function defined on the metric space $\Sigma(m)$. Henceforth the sets $E \in \Sigma$ will be considered as equivalence classes, namely, points of $\Sigma(m)$. The distance in $\Sigma(m)$ is denoted by $\rho$, given by $\rho(E, F)=m(E \triangle F)$. By (iii), $\rho(A, B)>0$, and the subset $\Sigma_{A, B}(m)=\{E: A \subseteq E \subseteq B\}$ is a closed in $\Sigma(m)$. Therefore, the restriction $\nu: \Sigma_{A, B}(m) \rightarrow \mathbb{R}$ is an upper semicontinuous function, bounded from above and defined on the complete metric space $\Sigma_{A, B}(m)$. Define on $\Sigma_{A, B}(m)$ the new function

$$
\widetilde{\nu}(X)=\nu(X)-\nu(A)-\frac{\nu(B)-\nu(A)}{m(B)-m(A)}(m(X)-m(A))
$$

for all $X \in \Sigma_{A, B}(m)$. Clearly, $\widetilde{\nu}(A)=\widetilde{\nu}(B)=0$. Set

$$
\sup _{X \in \Sigma_{A, B}(m)} \widetilde{\nu}(X)=a<+\infty
$$

We treat separately the two cases $a=0$ and $a>0$.

Case 1. If $a=0$, the maximum is attained at $A$. The first order condition $d^{+} \widetilde{\nu}(A)(B \backslash A) \leq 0$ leads easily to $d^{+} \nu(A)(B \backslash A) \leq \nu(B)-\nu(A)$, which manifestly implies (5.1) for any $\varepsilon>0$, by setting $C_{\varepsilon}=A$ and $\alpha_{\varepsilon}=1$. Note that also eq. (5.2) is trivially true by setting $C_{\varepsilon}=A$ and $\alpha_{\varepsilon}=1$.

Clearly, the maximum is attained at $B$ as well. Therefore, if the inner derivatives exist, we have also $d^{-} \nu(B)(B \backslash A) \geq \nu(B)-\nu(A)$ which yields (5.2) with $C_{\varepsilon}=B$ and $\beta_{\varepsilon}=0$. This way, (5.1) holds trivially as well.

Case 2. Assume $a>0$. We operate always in the following the restriction: $0<\varepsilon<a$. By Ekeland's variational principle [8] there exists a set $A \subseteq C_{\varepsilon} \subseteq B$ such that:

i) $\widetilde{\nu}\left(C_{\varepsilon}\right) \geq a-\varepsilon$ 
ii) $C_{\varepsilon}$ maximizes the function $\widetilde{\nu}(X)-\varepsilon^{1 / 2} \rho\left(X, C_{\varepsilon}\right)$ over $\Sigma_{A, B}(m)$.

As first thing, we show that the sets $C_{\varepsilon}$, as $\varepsilon$ varies, are uniformly away from $A$ and $B$ respectively. From the assumption $\varepsilon<a$, it follows that $\rho\left(A, C_{\varepsilon}\right)>0$ for all $\varepsilon$. We prove that $\rho\left(A, C_{\varepsilon}\right) \geq k>0$. Suppose not. There would then exist a sequence $\varepsilon_{n} \rightarrow 0$ such that $\rho\left(A, C_{\varepsilon_{n}}\right) \rightarrow 0$. By upper semicontinuity, $\limsup _{n} \widetilde{\nu}\left(C_{\varepsilon_{n}}\right) \leq \widetilde{\nu}(A)$. By (1), $\widetilde{\nu}\left(C_{\varepsilon_{n}}\right) \geq a-\varepsilon_{n} \Longrightarrow \lim \sup _{n} \widetilde{\nu}\left(C_{\varepsilon_{n}}\right) \geq a$. Hence, $\widetilde{\nu}(A) \geq a$, a contradiction. The same argument applies, by replacing $A$ by $B$. Consequently, $\rho\left(B, C_{\varepsilon}\right) \geq k^{\prime}>0$ for all $\varepsilon$ and for some $k^{\prime}$.

Next we exploit condition (ii) above. By the first order condition, $d^{+} \varphi\left(C_{\varepsilon}\right)\left(B \backslash C_{\varepsilon}\right) \leq$ 0 holds and $d^{-} \varphi\left(C_{\varepsilon}\right)\left(C_{\varepsilon} \backslash A\right) \geq 0$, with $\varphi(X)=\widetilde{\nu}(X)-\varepsilon^{1 / 2} \rho\left(X, C_{\varepsilon}\right)=\widetilde{\nu}(X)-$ $\varepsilon^{1 / 2} m\left(X \Delta C_{\varepsilon}\right)$. A straightforward computation of these derivatives leads to

$$
\begin{aligned}
& d^{+} \nu\left(C_{\varepsilon}\right)\left(B \backslash C_{\varepsilon}\right) \leq \rho\left(B, C_{\varepsilon}\right) \rho(A, B)^{-1}[\nu(B)-\nu(A)]+\varepsilon^{1 / 2} m\left(B \backslash C_{\varepsilon}\right) \\
& d^{-} \nu\left(C_{\varepsilon}\right)\left(C_{\varepsilon} \backslash A\right) \geq \rho\left(C_{\varepsilon}, A\right) \rho(A, B)^{-1}[\nu(B)-\nu(A)]-\varepsilon^{1 / 2} m\left(C_{\varepsilon} \backslash A\right) .
\end{aligned}
$$

Setting $\alpha_{\varepsilon}=\rho\left(B, C_{\varepsilon}\right)[\rho(A, B)]^{-1}$ and observing that $\rho\left(B, C_{\varepsilon}\right)+\rho\left(C_{\varepsilon}, A\right)=\rho(A, B)$, the desired result obtains.

Proposition 5.2. An implication is obvious. Suppose then that $d^{+} \nu(E) \geq 0$ for all $E$. Let $A \subseteq B$ and $m$ be the control measure. If $m(A)=m(B)$, then $\nu(A)=\nu(B)$. Assume hence that $m(B)>m(A)$. By Theorem 5.1,

$$
0 \leq d^{+} \nu\left(C_{\varepsilon}\right)\left(B \backslash C_{\varepsilon}\right) \leq \alpha_{\varepsilon}[\nu(B)-\nu(A)]+\varepsilon^{1 / 2} \rho\left(B, C_{\varepsilon}\right),
$$

for all $\varepsilon$ small enough. Since $\alpha_{\varepsilon} \in[k, 1]$, given a sequence $\varepsilon_{n} \rightarrow 0$, there is a subsequence, still denoted $\varepsilon_{n}$, such that $\alpha_{\varepsilon_{n}} \rightarrow \bar{\alpha}>0$. From $0 \leq \alpha_{\varepsilon_{n}}[\nu(B)-\nu(A)]+\varepsilon_{n}^{1 / 2} \rho\left(B, C_{\varepsilon_{n}}\right)$ and taking the limit, we get $\nu(B) \geq \nu(A)$.

Proposition 5.3. Set $\nu=\nu_{1}-\nu_{2}$. Fix $A$ and apply Theorem 5.1 with $B=\Omega$. We get

$$
0=d^{+} \nu\left(C_{\varepsilon}\right)\left(C_{\varepsilon}^{c}\right) \leq \alpha_{\varepsilon}[\nu(\Omega)-\nu(A)]+\varepsilon^{1 / 2} m\left(C_{\varepsilon}^{c}\right),
$$

with $\alpha_{\varepsilon} \in[k, 1]$. By the same argument adopted in Proposition 5.2, it follows that $\nu(A) \leq \nu(\Omega)$. Replacing $\nu$ by $-\nu$, in view of (5.3), yields

$$
0=d^{+} \nu\left(D_{\varepsilon}\right)\left(D_{\varepsilon}^{c}\right) \geq \alpha_{\varepsilon}^{\prime}[\nu(\Omega)-\nu(A)]-\varepsilon^{1 / 2} m\left(D_{\varepsilon}^{c}\right),
$$

that leads to $\nu(A) \geq \nu(\Omega)$. Namely, $\nu$ is constant. The other equivalence is similar.

Proposition 5.4. Set $\Delta^{+} \nu(A)(H)=D^{+} \nu(A)(H)+\sigma_{1}(H)$ and $\Delta^{+} \lambda(A)(H)=D^{+} \lambda(A)(H)+\sigma_{2}(H)$. Tedious but straightforward computations lead to the decomposition

$$
\begin{aligned}
\Delta^{+}(\nu \lambda)(A)= & \nu(A) D^{+} \lambda(A)+\lambda(A) D^{+} \nu(A)+\sigma_{1} D^{+} \lambda(A) \\
& +\nu(A \cup \cdot) \sigma_{2}+\lambda(A) \sigma_{1}+D^{+} \nu(A) D^{+} \lambda(A) .
\end{aligned}
$$


By assumption, $\left|\sigma_{1}\right| \rightarrow_{\pi} 0,\left|\sigma_{2}\right| \rightarrow_{\pi} 0$. By (i) of Proposition 3.3, $D^{+} \nu(A)$ and $D^{+} \lambda(A)$ are in $b a\left(\mathcal{A}_{A^{c}}\right)$. It follows that $\sigma_{1}(\cdot) D^{+} \lambda(A)(\cdot) \rightarrow_{\pi} 0$, $\nu(A \cup \cdot) \sigma_{2}(\cdot) \rightarrow_{\pi} 0$ and $\lambda(A) \sigma_{1}(\cdot) \rightarrow_{\pi} 0$ absolutely. Therefore it remains to study the refinement limit of $D^{+} \nu(A)(\cdot) D^{+} \lambda(A)(\cdot)$. Assume that $D^{+} \nu(A)$ is strongly continuous. Given an $\varepsilon>0$, there is a partition $\pi^{0} \in \Pi\left(A^{c}\right)$ such that $\left|D^{+} \nu(A)\right|\left(H_{i}\right) \leq \varepsilon$ for all $H_{i} \in \pi \succeq \pi^{0}$. Hence,

$$
\sum_{H_{i} \in \pi}\left|D^{+} \nu(A)\left(H_{i}\right) D^{+} \lambda(A)\left(H_{i}\right)\right| \leq \varepsilon \sum_{H_{i} \in \pi}\left|D^{+} \lambda(A)\left(H_{i}\right)\right| \leq \varepsilon M,
$$

where $M$ is the total variation of $D^{+} \lambda(A)$. This implies that $D^{+} \nu(A)(\cdot) D^{+} \lambda(A)(\cdot)$ converges to zero absolutely and, in turn, the claim. The statement for the inner derivatives can be achieved via dual set functions.

In order to prove Theorem 5.2 we formulate the following Lemma.

Let $\Sigma$ be a $\sigma$-algebra of subsets of a given space $\Omega$ and $\lambda$ be a symmetric $\sigma$-additive and finite measure on $(\Omega \times \Omega, \Sigma \otimes \Sigma)$ (i.e., $\lambda(A \times B)=\lambda(B \times A))$. Define the game $\nu(A)=\lambda(A \times A)$, for $A \in \Sigma$. Consider the marginal measure $\lambda_{m}(A)=\lambda(A \times \Omega)$ as well as the diagonal measure $\lambda_{d}(A)=\lambda((A \times \Omega) \cap \Delta)$.

Lemma 8.1. The game $\nu(A)=\lambda(A \times A)$ is $2\left|\lambda_{m}\right|$-Lipschitz. It is everywhere differentiable iff it is differentiable at $\varnothing$ and

$$
\begin{aligned}
& D^{+} \nu(A)=2 \lambda(A \times \cdot)+D^{+} \nu(\varnothing) \\
& D^{-} \nu(A)=2 \lambda(A \times \cdot)-D^{+} \nu(\varnothing) .
\end{aligned}
$$

In addition, if $\Omega$ is a Polish space and $\Sigma$ is its Borel $\sigma$-algebra, then $D^{+} \nu(\varnothing)$ exists and $D^{+} \nu(\varnothing)=\lambda_{d}$.

Proof. That $\nu$ is Lipschitz is left to the reader. Observe that $\bar{\nu}=2 \lambda_{m}-\nu$, therefore if $\nu$ is outer differentiable, then it is inner differentiable as well, and $d^{-} \nu(A)=$ $2 \lambda_{m}-d^{+} \nu\left(A^{c}\right)$. On the other hand, we have

$$
\Delta^{+} \nu(A)(H)=2 \lambda(A \times H)+\Delta^{+} \nu(\varnothing)(H) .
$$

Hence, $D^{+} \nu(A)(H)=2 \lambda(A \times H)+D^{+} \nu(\varnothing)(H)$.

Assume that $\Omega$ is a Polish space and $\lambda$ is positive. As $\lambda$ is tight, there is a compact $K \subset \Omega$ such that $\lambda(\Omega \times \Omega)-\lambda(K \times K) \leq \varepsilon$. Further, as $\lambda$ is regular, there is an open set $A \supseteq \Delta \cap(K \times K)$ such that $\lambda(A)-\lambda[\Delta \cap(K \times K)] \leq \varepsilon$. By compactness of $\Delta \cap(K \times K)$ it is easy to construct a partition $\pi=\left\{H_{i}\right\}_{i=1}^{n}$ of $K$ such that $H_{i} \in \pi \Longrightarrow$ $H_{i} \times H_{i} \subseteq A$. Consider the partition $\pi^{0}$ of $\Omega$ given by $\pi^{0}=\left\{H_{1}, \ldots, H_{n}, \Omega \backslash K\right\}$. Let 
$\pi=\left\{H_{i}^{\prime}, K_{j}\right\} \succeq \pi^{0}$ where $\left\{H_{i}^{\prime}\right\}$ is a partition of $K$, and $\left\{K_{j}\right\}$ is a partition of $\Omega \backslash K$.

We have

$$
\begin{aligned}
& \sum_{i}\left|\lambda\left(H_{i}^{\prime} \times H_{i}^{\prime}\right)-\lambda_{d}\left(H_{i}^{\prime}\right)\right|+\sum_{j}\left|\lambda\left(K_{j} \times K_{j}\right)-\lambda_{d}\left(K_{j}\right)\right| \\
\leq & \sum_{i} \lambda\left(H_{i}^{\prime} \times H_{i}^{\prime}\right)-\lambda\left(\left(H_{i}^{\prime} \times H_{i}^{\prime}\right) \cap \Delta\right)+\sum_{j} \lambda\left(K_{j} \times K_{j}\right) \\
\leq & \lambda\left(\cup_{i}\left(H_{i}^{\prime} \times H_{i}^{\prime}\right)\right)-\lambda(\Delta \cap[K \times K])+\varepsilon \\
\leq & \lambda(A)-\lambda[\Delta \cap(K \times K)]+\varepsilon \leq 2 \varepsilon .
\end{aligned}
$$

Hence, $D^{+} \nu(\varnothing)=\lambda_{d}$, provide $\lambda$ is positive. By the decomposition $\lambda=\lambda^{+}-\lambda^{-}$we get the desired result for any $\lambda$.

Theorem 5.2. Begin first with games of the type $\nu=\mu_{1} \cdot \mu_{2}$, with $\mu_{1}, \mu_{2} \in c a(\Sigma)$. Use Lemma 8.1 with the symmetric measure $\lambda=2^{-1}\left(\mu_{1} \otimes \mu_{2}+\mu_{2} \otimes \mu_{1}\right)$ on the product space $(\Omega \times \Omega, \Sigma \otimes \Sigma)$. $\nu$ turns out to be differentiable and

$$
D^{+} \mu_{1} \cdot \mu_{2}(\varnothing)=\left(\mu_{1} \otimes \mu_{2}\right)_{\Delta}
$$

Assume now that $\nu$ and $\lambda$ are games for which $D^{+} \nu(A)$ and $D^{+} \lambda(A)$ exist. By (i) and (ii) of Proposition 3.3, $D^{+} \nu(A)$ and $D^{+} \lambda(A)$ are in $c a\left(\Sigma_{A^{c}}\right)$. Use the same decomposition (8.3) as in the proof of Proposition 5.4. By assumption, $\left|\sigma_{1}\right| \rightarrow_{\pi} 0,\left|\sigma_{2}\right| \rightarrow_{\pi} 0$. Hence, as $D^{+} \lambda(A)$ and $\nu(A \cup \cdot)$ are bounded, it follows that $\sigma_{1} D^{+} \lambda(A), \nu(A \cup \cdot) \sigma_{2}$ and $\lambda(A) \sigma_{1}$ go to zero absolutely on $\Sigma_{A^{c}}$, while for the last term the limit is given by (8.4).

Proposition 5.5. Call $D^{+} \nu(A)=\mu_{1}, D^{+} \lambda(A)=\mu_{2}$ and $\left(\mu_{1} \otimes \mu_{2}\right)_{\Delta}$ the diagonal measure. With no loss of generality suppose $\mu_{1}$ and $\mu_{2}$ are non negative. By Fubini's theorem

$$
\left(\mu_{1} \otimes \mu_{2}\right)_{\Delta}(H)=\int_{H} \mu_{1}(s) d \mu_{2}(s),
$$

where the function $s \rightarrow \mu_{1}(s)$ is measurable. On the other hand, $\mu_{1}(s) \neq 0$ at most at countably many points which are the atoms of $\mu_{1}$. We get thus the representation as a summable series. Clearly $\left(\mu_{1} \otimes \mu_{2}\right)_{\Delta}$ is purely atomic.

Corollary 5.1. The games $q \circ \lambda$ are differentiable by Proposition 4.2. Relations (5.6) may be demonstrated by induction with the aid of differentiation rule of Theorem 5.2. Any monomial $x_{1} x_{2} \ldots x_{n}$ can be represented as

$$
\begin{aligned}
n x_{1} x_{2} \ldots x_{n}= & \left(x_{1}+\ldots+x_{n}\right)^{n}-\sum_{i}\left(x_{1}+\ldots+x_{n}-x_{i}\right)^{n} \\
& +\sum_{i, j}\left(x_{1}+\ldots+x_{n}-x_{i}-x_{j}\right)^{n}-\ldots \ldots \ldots
\end{aligned}
$$


(see [1, p. 41]). Therefore (5.6) implies the remaining statement.

Proposition 6.2. From Proposition 6.1 we know that if $d^{-} u(A)(A)=\nu(A)$ for all $A$, then $d^{-} u^{E}(A)(A)=\nu^{E}(A)$. If $E$ is a carrier of $\nu$, then $\nu^{E}=\nu$. The uniqueness of the potential implies that $u^{E}=u$. Hence $E$ is a carrier of $u$. Conversely, from $u^{E}=u$ we get $\nu^{E}=\nu$. The remaining of the proof is plain. Note that this implies $\mathcal{N}(u)=\mathcal{N}(\nu)$.

Proposition 6.4. The claim is proved by induction. Clearly, $P(\lambda)=\lambda$. Suppose to have proved that $P\left(\lambda^{s}\right)$ exist for any integer $s<n$ and for all $\lambda \in c a$, and that $P\left(\lambda^{s}\right)$ is a polynomial of the measures $\lambda,\left(\otimes^{2} \lambda\right)_{\Delta},\left(\otimes^{3} \lambda\right)_{\Delta}, \ldots,\left(\otimes^{s} \lambda\right)_{\Delta}$. This implies the existence of potentials of any monomial $\lambda_{1} \lambda_{2} \ldots \lambda_{s}$ for any integer $s<n$. This is a consequence of the representation (8.5). Consider now the game $\lambda^{n}$. It is differentiable and by (5.6) the derivative is

$$
\begin{aligned}
D^{-} \lambda^{n}(A)(A)= & n \lambda^{n}(A)-\left(\begin{array}{l}
n \\
2
\end{array}\right) \lambda^{n-2}(A)\left(\otimes^{2} \lambda\right)_{\Delta}(A) \\
& +\left(\begin{array}{l}
n \\
3
\end{array}\right) \lambda^{n-3}(A)\left(\otimes^{3} \lambda\right)_{\Delta}(A)-\ldots \ldots
\end{aligned}
$$

Therefore

$$
\begin{aligned}
P\left(\lambda^{n}\right)= & n^{-1} \lambda^{n}+n^{-1}\left(\begin{array}{l}
n \\
2
\end{array}\right) P\left[\lambda^{n-2}\left(\otimes^{2} \lambda\right)_{\Delta}\right] \\
& -n^{-1}\left(\begin{array}{l}
n \\
3
\end{array}\right) P\left[\lambda^{n-3}\left(\otimes^{3} \lambda\right)_{\Delta}\right]+\ldots
\end{aligned}
$$

In the right hand we have to compute potentials of monomials of degree less or equal to $n-1$. We conclude that $P\left(\lambda^{n}\right)$ exists for any integer $n$ and they are polynomials. Relation (8.5) extends this result to any polynomial $q(\lambda)$.

Lemma 6.1. Step 1. Let $\nu(A)=d^{-} u(A)(A)$. We prove that

$$
d^{-} \nu(A)(H)=d_{2}^{--} u(A)(A, H) \text {. }
$$

Actually,

$$
\begin{aligned}
d^{-} \nu(A)(H) & =\lim _{\pi \in \Pi(H)} \sum_{H_{i} \in \pi} d^{-} u(A)(A)-d^{-} u\left(A \backslash H_{i}\right)\left(A \backslash H_{i}\right) \\
& =\lim _{\pi \in \Pi(H)} \sum_{H_{i} \in \pi} d^{-} \widetilde{u}(A)(A)-d^{-} \widetilde{u}\left(A \backslash H_{i}\right)(A) \\
& =d_{2}^{--} u(A)(A, H) .
\end{aligned}
$$

Step 2. From (8.6) and by the symmetry of the second derivative, $d^{-} \nu(A)(H)=d_{2}^{--} u(A)(H, A)$. By setting $\varphi_{H}(A)=d^{-} u(A)(H)$, we get $d^{-} \nu(A)(H)=$ 
$d^{-} \varphi_{H}(A)(A)$ for all $A$ and all $H \subseteq A$. If $\nu$ is monotone, then $d^{-} \nu(A)(H) \geq 0$ which implies that $d^{-} \varphi_{H}(A)(A) \geq 0$ for all $A$. An application of mean value theorem yields $\varphi_{H}(A)=d^{-} u(A)(H) \geq 0$ for all $A$. As $u \in \mathcal{C}(\Sigma)$, Proposition 5.2 implies $u$ is monotone.

Theorem 6.1. The proof is divided into several steps.

Step 1. Any game $\nu=p \circ \lambda \in$ pol $M$ is regularly inner twice differentiable. The proof of this is lengthy. Indeed, in this proof it is enough to check it, provided each measure $\lambda_{i}$ has finitely many atoms. In this case, we can then operate the decomposition (see for instance [15]) of each measure $\lambda_{i}$ as $\lambda_{i}=\mu_{i}+\sum_{r=1}^{k} a^{i} \delta_{r}$, where $\mu_{i}$ are nonatomic measures, $a^{i}$ are scalars, $\delta_{r}$ is the Dirac measure concentrated at the point $\left\{s_{r}\right\}$ and $S=\left\{s_{1}, \ldots, s_{k}\right\}$ is the totality of atoms of all $\lambda_{i}$. It follows easily the representation

$$
p(\lambda)=p(\mu)+\sum_{\varnothing \neq T \subseteq S} p_{T}(\mu) u_{T}
$$

where $u_{T}$ are unanimity games, $\mu=\left(\mu_{i}\right)_{i=1}^{n}$ and $p_{T}(x)$ are appropriate polynomials of degrees $\operatorname{deg}(p)-|T|$. By iterating twice the multiplicative rule of Proposition 5.4 it easy to check that all these addenda $p_{T}(\mu) u_{T}$ are regularly inner twice differentiable and hence $p(\lambda)$ is.

Step 2. Any $\nu \in \operatorname{pol} M$ satisfies condition (ii) of Lemma 6.1. Actually, if $\nu=\lambda^{n}$, formula (5.6) furnishes

$$
D^{-} \nu(A)(H)=n \lambda^{n-1}(A) \lambda^{H}(A)-\left(\begin{array}{c}
n \\
2
\end{array}\right) \lambda^{n-2}(A)\left(\otimes^{2} \lambda\right)_{\Delta}^{H}(A)+\ldots \ldots
$$

which is a polynomial game for the measures $\lambda, \lambda^{H},\left(\otimes^{2} \lambda\right)_{\Delta}^{H}, \ldots .$. . Therefore $A \rightarrow$ $D^{-} \nu(A)(H)$ is measure continuous as it is Lipschitz. This result may be extended to any product of measures $\lambda_{1} \lambda_{2} \ldots \lambda_{n}$ by the usual method.

Step 3. We prove now that $P$ preserves monotonicity. Let $\nu=p \circ \lambda$ where $\lambda=$ $\left(\lambda_{1}, \ldots, \lambda_{n}\right)$ are generic finite measures. By Proposition 6.4 the potential $u=P \nu$ exists and is a polynomial game. Let $S=\left\{s_{1}, s_{2}, \ldots ..\right\}$ be the set of atoms of all the measures $\lambda_{i}$. Set $S_{n}=\left\{s_{n}, s_{n+1}, \ldots ..\right\}$ and consider the sequence of games $\nu^{n}=\nu^{S_{n}^{c}}=p \circ \lambda^{S_{n}^{c}}$. We have that $P \nu^{n}=u^{n}=u^{S_{n}^{c}}$. By Proposition 6.4 and 6.2 the games $u^{n}$ are polynomials of measures with finitely many atoms. Suppose that $\mu \succeq \nu^{n} \succeq-\mu$. It follow that $\nu^{n}+\mu$ is monotone with potential given by $u^{n}+\mu$. In view of steps $1-2, u^{n}+\mu$ satisfies conditions (i)-(ii) of Lemma 6.1. Hence $u^{n}+\mu$ is monotone. The same argument applies for $\mu-\nu^{n}$. We deduce that $\left\|u^{n}\right\|_{\infty} \leq\left\|\nu^{n}\right\|_{\infty}$ for all $n$. As $\left\|\nu-\nu^{n}\right\|_{\infty} \rightarrow 0$, it is easy to check that $\left\|u^{n}-u\right\|_{\infty} \rightarrow 0$. To conclude, $u$ is monotone whenever $\nu=p \circ \lambda$ is monotonic.

Step 4. Let $\nu \in p M_{\infty}$. We prove that $P \nu$ exists and lies in $p M_{\infty}$. Let $\left\|\nu_{n}-\nu\right\|_{\infty} \rightarrow 0$ with $\nu_{n}$ polynomial games. By Step 3, $\left\|P \nu_{n}\right\|_{\infty} \leq\left\|\nu_{n}\right\|_{\infty}$. It follows that the sequence 
$u_{n}=P \nu_{n}$ is Cauchy and hence $u_{n} \rightarrow u \in p M_{\infty}$. From $D^{-} u_{n}(A)(A)=\nu_{n}(A)$ and Theorem 4.2, we get $D^{-} u(A)(A)=\nu(A)$ and $u$ is the potential of $\nu$.

Step 5. It remains to prove that $P \nu$ preserves monotonicity when $\nu \in p M_{\infty}$. According to the definition of the measures $\nu^{*}$ and $\nu_{*}$ (see Preliminary Section) it is easy to check (see [22]) that for any game $\nu \in \mathcal{L}(\Sigma)$ we have $\nu^{*}, \nu_{*} \in c a(\Sigma),\left(\nu_{1}+\nu_{2}\right)_{*} \geq\left(\nu_{1}\right)_{*}+\left(\nu_{2}\right)_{*}$ and $\|\nu\|_{\infty}=\left\|\left|\nu^{*}\right| \vee\left|\nu_{*}\right|\right\|_{b v}$. This last property entails that $\left\|\nu_{*}\right\|_{b v} \leq\|\nu\|_{\infty}$. Clearly $\nu$ is monotone iff $\nu_{*} \geq 0$.

Let $\nu^{n} \rightarrow \nu$ in $p M_{\infty}$ where $\nu^{n}$ is a sequence of polynomial games. The relation $\nu^{n}=\left(\nu^{n}-\nu\right)+\nu$ implies $\nu_{*}^{n} \geq\left(\nu^{n}-\nu\right)_{*}+\nu_{*}$. As $\nu^{n} \succeq \nu_{*}^{n}$, we have $\nu^{n} \succeq\left(\nu^{n}-\nu\right)_{*}+\nu_{*}$. By the monotonicity property of the operator $P$ valid for polynomial games, we get $u^{n} \succeq\left(\nu^{n}-\nu\right)_{*}+\nu_{*}$ where $u^{n}=P \nu^{n}$. As $\left\|\nu^{n}-\nu\right\|_{\infty} \rightarrow 0$, we get $u \succeq \nu_{*}$. Therefore $u$ is monotone, provided $\nu$ is monotone.

Proposition 7.1. We prove only the consistency of $\psi$. The remaining claims are obvious. Consistency is discussed at length in [12]. Following Hart and Mas-Colell's definition, if $\phi$ is an efficient solution defined on a restrictable class of games, and $T$ is a fixed coalition, define the reduced game of $\nu$ as $\nu_{T}^{\phi}(S)=\phi\left(\nu^{S \cup T^{c}}\right)(S)$ for all $S \subseteq T$ (see eq. (4.3) of [12]). Notice that $\nu_{T}^{\phi}(S)$ is a game defined on $\Sigma_{T}$. The solution $\phi$ is called consistent provided $\phi \nu=\phi \nu_{T}^{\phi}$ holds over $T$ and for all $T \in \Sigma$.

If $P \nu=u$, then $P \nu^{S \cup T^{c}}=u^{S \cup T^{c}}$. It follows that $\nu_{T}^{\psi}(S)=d^{-} u\left(S \cup T^{c}\right)(S)$. Therefore the set function $u_{1}(\cdot)=u\left(\cdot \cup T^{c}\right)$ is a potential of the game $\nu_{T}^{\psi}$. Note that $u_{1}(\cdot)$ is not a game. The normalized potential would be $u_{1}(\cdot)-u\left(T^{c}\right)$. To conclude, for $H \subseteq T$,

$$
\psi\left(\nu_{T}^{\psi}\right)(H)=d^{-} u_{1}(T)(H)=d^{-} u(\Omega)(H)=\psi \nu(H)
$$

which is the desired result.

Theorem 7.1. Step 1. By Theorem $6.1 p M_{\infty} \subset$ POT. To prove that $\psi$ is a value, by Proposition 7.1 it suffices to verify that $\psi$ is a positive operator. Assume $\nu \in p M_{\infty}$ is monotone. By Theorem 6.1 its potential $u=P \nu$ is a monotone game in $p M_{\infty}$. Hence $\psi \nu=D^{-} u(\Omega) \geq 0$ and $\psi$ is positive. The other properties of $\psi$ follow straightforwardly (see $[22])$.

Step 2. Let now $\varphi: p M_{\infty} \rightarrow b a$ be any value operator. By its positivity, it satisfies the Milnor condition (7.1). This in turn entails that $\varphi$ is norm-continuous and takes values on $c a$. Clearly the set of polynomial games $p \circ \lambda$, with measures $\lambda$ having finitely many atoms only, is dense in $p M_{\infty}$. Therefore, in view of the argument discussed in step 1 of the proof of Theorem $6.1, \varphi$ is uniquely determined by its values at the games $\lambda^{n} u_{T}$, with $\lambda \in n a^{1}$ and $u_{T}$ are unanimity games with $|T|<\infty$.

Step 3. By symmetry of $\varphi$, we prove that necessarily

$$
\varphi\left(\lambda^{n} u_{T}\right)(H)=\alpha_{n, t} \lambda(H)+\left(1-\alpha_{n, t}\right) \frac{|H \cap T|}{|T|}
$$


where $\alpha_{n, t}$ are a nonnegative scalars, depending on $n$ and $t=|T|$. Notice that $\psi\left(\lambda^{n} u_{T}\right)$ are given in (7.2) and are a specialization of the representation (8.7).

Fix $\nu=\lambda^{n} u_{T}$ and consider the automorphisms group

$$
G(\nu)=\left\{\vartheta: \vartheta^{*} \lambda=\lambda \text { and } \vartheta T=T\right\} .
$$

$G(\nu)$ leaves $\nu$ as fixed point, i.e., $\vartheta^{*} \nu=\nu$ for all $\vartheta \in G(\nu)$. Set $\varphi\left(\lambda^{n} u_{T}\right)=\mu$. There is a unique decomposition $\mu=\mu_{n a}+\mu_{p}$ where $\mu_{n a}$ is its nonatomic component and $\mu_{p}$ is the purely atomic part. If $\vartheta \in G(\nu), \vartheta^{*} \mu=\vartheta^{*} \mu_{n a}+\vartheta^{*} \mu_{p}=\mu_{n a}+\mu_{p}$. By uniqueness of the decomposition, it follows that $\vartheta^{*} \mu_{n a}=\mu_{n a}$ and $\vartheta^{*} \mu_{p}=\mu_{p}$ for all $\vartheta \in G(\nu)$. By standard argument (see remark below), $\mu_{n a}=\alpha \lambda$. If $a$ is an atom of $\mu_{p}$, we obtain that $\mu_{p}(a)=\mu_{p}(\vartheta a)$. This implies in turn that the carrier of $\mu_{p}$ is $T$. Further, the measure $\mu_{p}$ is uniform over $T$. Thus $\mu_{p}(H)=k|H \cap T|$ and $\varphi\left(\lambda^{n} u_{T}\right)=\alpha \lambda+k|\cdot \cap T|$. By efficiency and positivity of $\varphi$, we get the representation (8.7). We have still to show that $\alpha_{n, t}$ depends merely on $t=|T|$ rather than $T$. This is an easy exercise. If $|T|=|Q|$, consider an automorphism $\vartheta$ such that $\vartheta Q=T$ and $\vartheta^{*} \lambda=\lambda$. Under standardness condition such an automorphism does exist. Clearly, $\vartheta^{*}\left(\lambda^{n} u_{T}\right)=\lambda^{n} u_{Q}$. By symmetry of $\varphi$, it is then easy to show that the coefficient $\alpha_{n, t}$ in the representation (8.7) is identical for the two games $\lambda^{n} u_{T}$ and $\lambda^{n} u_{Q}$.

Step 4. Assume now that $\varphi$ is the asymptotic value on $p M_{\infty}$, necessarily existing by [25]. It suffices to prove that $\varphi\left(\lambda^{n} u_{T}\right)=\psi\left(\lambda^{n} u_{T}\right)$. By (8.7) and (7.2), it is enough to show that $\varphi\left(\lambda^{n} u_{T}\right)(T)=t(t+n)^{-1}$. Let $T=\left\{a_{1}, a_{2}, \ldots, a_{t}\right\}$, and consider the following $T$ feasible sequence of partitions of the space $\Omega, \pi_{m}=\left\{a_{1}, \ldots, a_{t}, H_{1}, H_{2}, \ldots, H_{m}\right\}$, where $\lambda\left(H_{i}\right)=1 / m$. As $\lambda$ is nonatomic, a partition $\pi_{m}$ with such requirements does exist. Set $\nu_{m}=\nu_{\pi_{m}}$. We aim at calculating $S h \nu_{m}(T)$. The multilinear extension of $\nu_{m}$ is

$$
B_{\nu_{m}}=\sum_{L}\left(\frac{|L|}{m}\right)^{n} x_{1} x_{2} \ldots x_{t} \prod_{i \in L} y_{i} \prod_{j \in L^{c}}\left(1-y_{j}\right)
$$

where the summation is made over all the subsets $L \subseteq\{1,2, \ldots, m\}$ and the variables associated with the atoms $a_{1}, \ldots, a_{t}$ and those associated with the atoms $H_{1}, H_{2}, \ldots, H_{m}$ are denoted differently. By Owen's diagonal formula it follows that

$$
S h \nu_{m}(T)=t \int_{0}^{1} s^{t-1}\left(\sum_{r=0}^{m}\left(\begin{array}{c}
m \\
r
\end{array}\right)\left(\frac{r}{m}\right)^{n} s^{r}(1-s)^{m-r}\right) d s .
$$

The functions

$$
\rho_{m}(s)=\sum_{r=0}^{m}\left(\begin{array}{c}
m \\
r
\end{array}\right)\left(\frac{r}{m}\right)^{n} s^{r}(1-s)^{m-r}
$$

are the Bernstein's polynomials associated with the function $s^{n}$. Thus $\rho_{m}(s) \rightarrow s^{n}$ as $m \rightarrow \infty$, uniformly on the interval $[0,1]$. This implies

$$
\varphi \nu(T)=\lim _{m \rightarrow \infty} S h \nu_{m}(T)=t \int_{0}^{1} s^{t+n-1} d s=t(n+t)^{-1},
$$


which proves the assertion.

As regarding the last statement, the value is unique over $p N A_{\infty}$. This is proven in [22] and [13]. Hence the restriction of $\psi$ to $p N A_{\infty}$ is the Aumann-Shapley value.

Remark. In Step 3 of the above Theorem, we used a rather standard argument in value theory (see proof of Proposition 6.1 of [1]). Let us further clarify this argument. Fix a coalition $A$ with $\lambda(A)>0$ and let $B$ be any coalition such the $\lambda(B)=\lambda(A)$. By Weiss' argument (see Note 1 on page 40 of [1]) there is an automorphism $\vartheta \in G(\nu)$ such that $\vartheta(A)=B$. This implies that $\mu_{n a}(A)=\mu_{n a}(B)$. This property suffices to infer that

$\mu_{n a}=\alpha \lambda$. See for instance Theorem 4 of [27]. Indeed a more general theorem based on this property can be found in [17] (Theorem 20).

Theorem 7.2. $P \nu=u$ implies $P\left(\vartheta^{*} \nu\right)=\vartheta^{*} u$. Further,

$$
d_{2}^{--}\left(\vartheta^{*} u\right)(A)(H, K)=d_{2}^{--} u(\vartheta A)(\vartheta H, \vartheta K)
$$

that implies the space $\mathrm{POT}_{2}(\Sigma)$ to be symmetric. An analogous argument applies to the restrictability in that

$$
d_{2}^{--}\left(u^{S}\right)(A)(H, K)=d_{2}^{--} u(A \cap S)(H \cap S, K \cap S) .
$$

Clearly $\psi$ is a positive operator by Theorem 6.1 and satisfies the balanced contribution axiom by definition.

\section{References}

[1] R.J. Aumann, L.S. Shapley, Values of non-atomic games. Princeton University Press, 1974.

[2] K.P.S.M. Bhaskara Rao, M. Bhaskara Rao, Theory of Charges, Academic Press, New York, 1983.

[3] J.M. Borwein, A.S. Lewis, Convex Analysis and Nonlinear Optimization, SpringerVerlag, New York, 2000.

[4] E. Calvo, J.C. Santos, Potentials in cooperative TU-games, Mathematical Social Sciences, 34, 175-190, 1997.

[5] J. Diestel, J.J. Uhl, Vector Measures, American Mathematical Society, Providence Rhode Island, 1977.

[6] N. Dunford, J.T. Schwartz, Linear operators, part I: general theory. WileyInterscience, London, 1954. 
[7] E. Einy, D. Monderer, D. Moreno, The least core, kernel and bargaining sets of large games, Economic Theory, 11, 585-601, 1998.

[8] I. Ekeland, On the variational principle, Journal of Mathematical Analysis and Applications, 47, 324-353, 1974.

[9] L.G. Epstein, A definition of uncertainty aversion, Review of Economic Studies, 66, 579-608, 1999.

[10] L.G. Epstein, M. Marinacci, The core of large differentiable TU games, Journal of Economic Theory, 100, 235-273, 2001.

[11] S. Hart, Values of mixed games, International Journal of Game Theory, 2, 69-85, 1973.

[12] S. Hart, A. Mas-Colell, Potential, Value and Consistency, Econometrica, 57, 589$614,1989$.

[13] S. Hart, D. Monderer, Potentials and weighted values of nonatomic games, Mathematics of Operations Research, 22, 619-630, 1997.

[14] J. Jahn, Introduction to the theory of nonlinear optimization, Springer-Verlag, Berlin,1996.

[15] R. A. Johnson, Atomic and Nonatomic Measures, Proceedings of the American Mathematical Society, 25, 650-655, 1970.

[16] Y. Kannai, Values of games with a continuum of players, Israel Journal of Mathematics, 4, 54-58, 1966.

[17] M. Marinacci, L. Montrucchio, Subcalculus for Set Functions and Cores of TU Games, Journal of Mathematical Economics, 39, 1-25, 2003.

[18] M. Marinacci, L. Montrucchio, Introduction to the Mathematics of Ambiguity, in Uncertainty in economic theory. (I. Gilboa, ed.), pp. 46-107, Routledge, London, 2004.

[19] M. Marinacci, L. Montrucchio, Ultramodular functions, Mathematics of Operations Research, forthcoming.

[20] M. Marinacci, L. Montrucchio, Stable Cores of Large Games, 2004. International Journal of Game Theory, forthcoming.

[21] J.F. Mertens, Values and derivatives, Mathematics of Operations Research, 5, 523$552,1980$. 
[22] D. Monderer, A Milnor condition for nonatomic Lipschitz games and its applications, Mathematics of Operations Research, 15, 714-723, 1990.

[23] D. Monderer, D. Samet, Variations on the Shapley value, in Handbook of Game Theory, Vol. 3, R. Aumann, S. Hart (eds.), pp. 2005-2076, North-Holland, Amsterdam, 2002.

[24] R. B. Myerson, Conference structure and fair allocation rules, International Journal of Game Theory, 9,169-182, 1980.

[25] A. Neyman, Singular games having asymptotic values, Mathematics of Operations Research, 6, 205-212, 1981.

[26] A. Neyman, Values of Games with Infinitely Many Players, In Handbook of Game Theory, Vol. 3, R. Aumann, S. Hart, eds., North-Holland, Amsterdam, 2002.

[27] J.E. Nymann, A uniqueness condition for finite measures, Proceedings of the American Mathematical Society, 108, 913-919, 1990.

[28] G. Owen, Multilinear extensions of games. Management Sciences 18, 64-79, 1972.

[29] J. Rosenmuller, Some properties of convex set functions, Operations Research, 2, 287-307, 1972.

[30] J. Rosenmuller, Extreme Games and Their Solutions, Springer-Verlag, Berlin, 1977.

[31] L.S. Shapley, A value for n-person games, in Contributions to the Theory of Games (H. Kuhn and A.W. Tucker, eds.), Princeton University Press, Princeton, 1953.

[32] D. Schmeidler, Cores of exact games, Journal of Mathematical Analysis and Applications, 40, 214-225, 1972. 